\title{
Metabarcoding monitoring analysis: the pros and cons of using co-extracted environmental DNA and RNA data to assess offshore oil production impacts on benthic communities
}

\author{
Olivier Laroche ${ }^{\text {Corresp., }}{ }^{1,2}$, Susanna A Wood ${ }^{2,3}$, Louis A Tremblay ${ }^{1,2}$, Gavin Lear ${ }^{1}$, Joanne I Ellis ${ }^{4}$, Xavier \\ Pochon ${ }^{2,5}$ \\ ${ }^{1}$ School of Biological Sciences, University of Auckland, Auckland, New Zealand \\ 2 Environmental Technologies, Coastal and Freshwater Group, Cawthron Institute, Nelson, New Zealand \\ 3 Environmental Research Institute, University of Waikato, Hamilton, New Zealand \\ 4 Red Sea Research Centre, King Abdullah University of Science and Technology, Thuwal, Saudi Arabia \\ 5 Institute of Marine Science, University of Auckland, Auckland, New Zealand \\ Corresponding Author: Olivier Laroche \\ Email address: olar785@aucklanduni.ac.nz
}

Sequencing environmental DNA (eDNA) is increasingly being used as an alternative to traditional morphological-based identification to characterize biological assemblages and monitor anthropogenic impacts in marine environments. Most studies only assess eDNA which, compared to eRNA, can persist longer in the environment after cell death. Therefore, eRNA may provide a more immediate census of the environment due to its relatively weaker stability, leading some researchers to advocate for the use of eRNA as an additional, or perhaps superior proxy for portraying ecological changes. A variety of pretreatment techniques for screening eDNA and eRNA derived operational taxonomic units (OTUs) have been employed prior to statistical analyses, including removing singleton taxa (i.e., OTUs found only once) and discarding those not present in both eDNA and eRNA datasets. In this study, we used bacterial (16S ribosomal RNA gene) and eukaryotic (18S ribosomal RNA gene) eDNA- and eRNA-derived data from benthic communities collected at increasing distances along a transect from an oil production platform (Taranaki, New Zealand). Macro-infauna (visual classification of benthic invertebrates) and physicochemical data were analyzed in parallel. We tested the effect of removing singleton taxa, and removing taxa not present in the eDNA and eRNA libraries from the same environmental sample (trimmed by shared OTUs), by comparing the impact of the oil production platform on alpha- and beta-diversity of the eDNA/eRNA-based biological assemblages, and by correlating these to the morphologically identified macro-faunal communities and the physico-chemical data. When trimmed by singletons, presence/absence information from eRNA data represented the best proxy to detect 
changes on species diversity for both bacteria and eukaryotes. However, assessment of quantitative beta-diversity from read abundance information of bacteria eRNA did not, contrary to eDNA, reveal any impact from the oil production activity. Overall, the data appeared more robust when trimmed by shared OTUs, showing a greater effect of the platform on alpha- and beta-diversity. Trimming by shared OTUs likely removes taxa derived from legacy DNA and technical artefacts introduced through reverse transcriptase, polymerase-chain-reaction and sequencing. Findings from our scoping study suggest that metabarcoding-based biomonitoring surveys should, if funds, time and expertise allow, be assessed using both eDNA and eRNA products. 


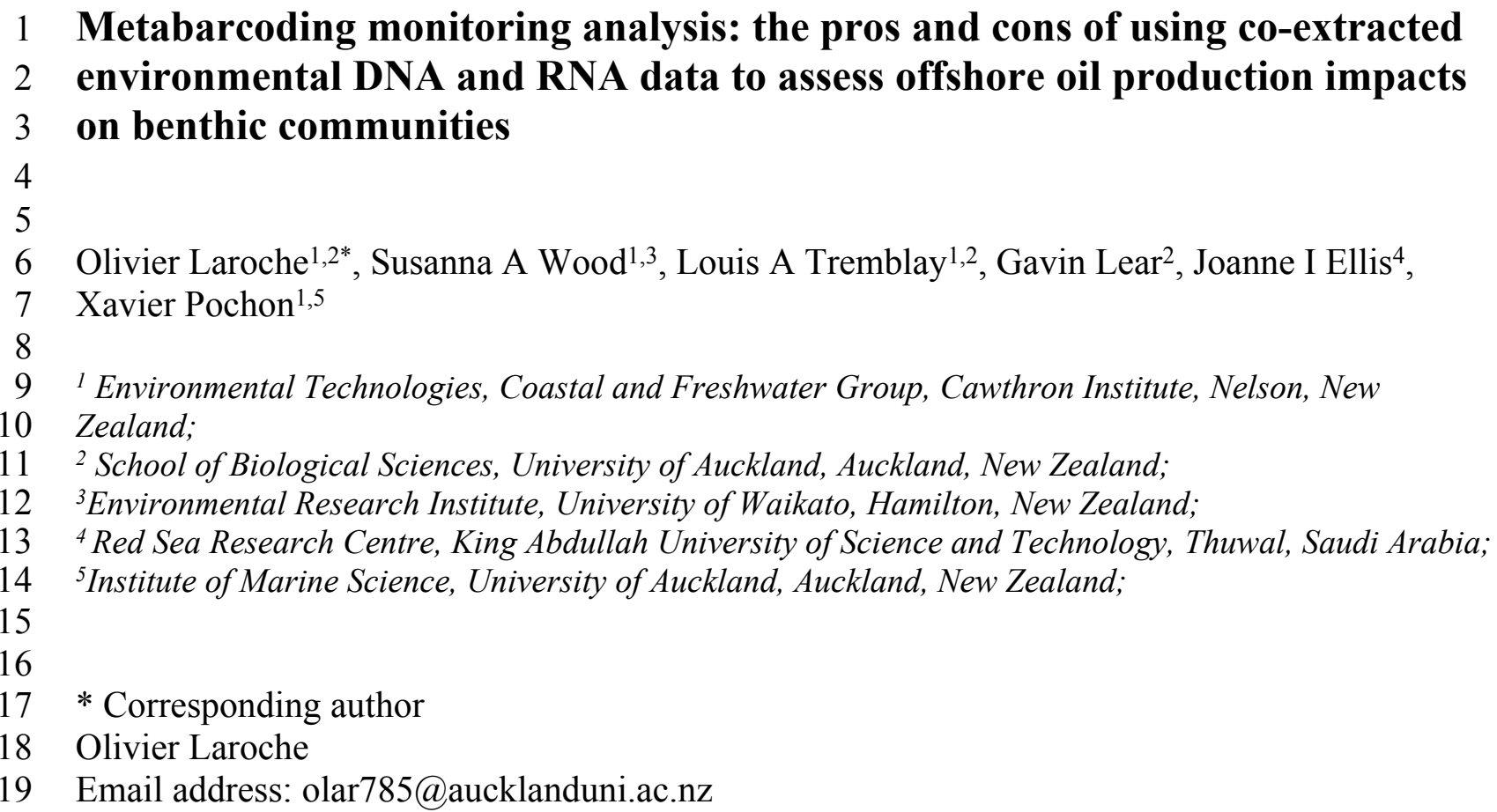




\section{Abstract}

Sequencing environmental DNA (eDNA) is increasingly being used as an alternative to traditional morphological-based identification to characterize biological assemblages and monitor anthropogenic impacts in marine environments. Most studies only assess eDNA which, compared to eRNA, can persist longer in the environment after cell death. Therefore, eRNA may provide a more immediate census of the environment due to its relatively weaker stability, leading some researchers to advocate for the use of eRNA as an additional, or perhaps superior proxy for portraying ecological changes. A variety of pre-treatment techniques for screening eDNA and eRNA derived operational taxonomic units (OTUs) have been employed prior to statistical analyses, including removing singleton taxa (i.e., OTUs found only once) and discarding those not present in both eDNA and eRNA datasets. In this study, we used bacterial (16S ribosomal RNA gene) and eukaryotic (18S ribosomal RNA gene) eDNA- and eRNAderived data from benthic communities collected at increasing distances along a transect from an oil production platform (Taranaki, New Zealand). Macro-infauna (visual classification of benthic invertebrates) and physico-chemical data were analyzed in parallel. We tested the effect of removing singleton taxa, and removing taxa not present in the eDNA and eRNA libraries from the same environmental sample (trimmed by shared OTUs), by comparing the impact of the oil production platform on alpha- and beta-diversity of the eDNA/eRNA-based biological assemblages, and by correlating these to the morphologically identified macro-faunal communities and the physico-chemical data. When trimmed by singletons, presence/absence information from eRNA data represented the best proxy to detect changes on species diversity for both bacteria and eukaryotes. However, assessment of quantitative beta-diversity from read abundance information of bacteria eRNA did not, contrary to eDNA, reveal any impact from the oil production activity. Overall, the data appeared more robust when trimmed by shared OTUs, showing a greater effect of the platform on alpha- and beta-diversity. Trimming by shared OTUs likely removes taxa derived from legacy DNA and technical artefacts introduced through reverse transcriptase, polymerase-chain-reaction and sequencing. Findings from our scoping study suggest that metabarcoding-based biomonitoring surveys should, if funds, time and expertise allow, be assessed using both eDNA and eRNA products. 
54

55

56

57

58

\section{Introduction}

Environmental DNA (eDNA) metabarcoding, defined here as the combined use of universal DNA barcodes and high-throughput sequencing (HTS) to characterize biological communities from genetic material collected from environmental samples (sediment, water, etc.) is increasingly being used to assess biodiversity and anthropogenic impacts in terrestrial (e.g. Yu et al., 2012; Taberlet et al., 2012; Ji et al., 2013; Beng et al., 2016) and aquatic environments (e.g. Carew et al., 2013; Chariton et al., 2014; Visco et al., 2015; Dowle et al., 2015; Pochon et al., 2015; Pawlowski et al., 2016; Abad et al., 2016; Lanzén et al., 2016). Metabarcoding is a cost-effective method that can rapidly and simultaneously target multiple species to complement traditional ecosystem biomonitoring approaches (Valentini, Pompanon \& Taberlet, 2009; Bourlat et al., 2013; Ji et al., 2013; Bohmann et al., 2014). It also allows microbiota that are difficult or impossible to identify morphologically to be characterized. Micro-organisms are at the bottom of the food chain, have shorter life cycles, and often have a higher diversity and abundance than macro-fauna. They may therefore be a better proxy for evaluating environmental impacts, and their use has been advocated for biomonitoring (Bourlat et al., 2013; Pawlowski et al., 2014, 2016b; Pawlowski, Lejzerowicz \& Esling, 2014; Lejzerowicz et al., 2015; Dowle et al., 2015; Lau et al., 2015).

Most metabarcoding monitoring studies use eDNA to characterize biological communities. A limited number have also evaluated data from co-extracted eRNA products (e.g. Pawlowski et al., 2014, 2016a; Visco et al., 2015; Dowle et al., 2015; Pochon et al., 2015; Laroche et al., 2016). Because RNA deteriorates rapidly after cell death, eRNA likely provides a more accurate representation of viable communities (Mengoni et al., 2005). Blazewicz et al. (2013) suggested that the relative concentration of RNA in the environment provides a robust indication of the growth and adaptation potential of microbial communities. In general, when 
environmental metabarcoding studies have used both eDNA and eRNA they have found slightly stronger correlations between community data generated from eRNA and environmental conditions (Pawlowski et al., 2014, 2016a; Visco et al., 2015; Dowle et al., 2015; Pochon et al., 2015; Laroche et al., 2016). However, working with eRNA adds additional complexities and expenses related to sampling and laboratory analysis including extra precaution during sampling, transport and storage to avoid degradation. A greater understanding of the potential benefits of using eDNA versus eRNA is therefore desirable prior to incorporating metabarcoding methods into environmental monitoring programs.

Previous studies assessing eDNA and eRNA extracted from the same environmental sample have noted differing diversities of operational taxonomic units (OTUs) recovered from assessment of either molecule. Greater taxon richness derived from eDNA data can be explained through the detection of DNA from dead organisms as well as extracellular DNA (free-floating or legacy DNA) that has bound to sediment or other particles. However, many studies report an important number of taxa only detected from eRNA (e.g. Pawlowski et al., 2014, 2016a; Pochon et al., 2015; Laroche et al., 2016; Hu et al., 2016). These OTUs may be the result of $\mathrm{PCR} /$ sequencing artefacts or the detection of rare but very active taxa. To date, most studies, have dealt with this by only retaining the data of taxa present in both eDNA/eRNA datasets across the entire sample set (Pawlowski et al., 2014, 2016a; Dowle et al., 2015; Pochon et al., 2015; Laroche et al., 2016), or within the same sites (Hu et al., 2016).

A further data treatment technique which is commonly applied to datasets, is the use of minimal DNA sequence read abundance thresholds for each unique taxon (i.e., data related to any particular OTU is discarded if insufficient numbers of that taxon are detected in the overall dataset, typically 2 to 10 reads). This is generally done to reduce sequencing artefacts (Hunt et 
101 al., 2013; Charvet, Vincent \& Lovejoy, 2014; Visco et al., 2015). The methods applied to the

102 pre-treatment of metabarcoding data (i.e., use of a minimum abundance removal threshold or

103 dataset trimming based on shared-taxa abundances) can have important implications on the final

104 resolution (number of available DNA sequence reads and/or taxa), sensitivity (smallest

105 perceptible effect) and accuracy (confidence level) of the data used to assess anthropogenic

106 impacts.

107 The aims of this study was to compare the use of co-extracted eDNA and eRNA products

108 to assess the benthic environment around an offshore oil production platform, and to determine

109 whether eDNA- or eRNA-derived taxa provide more accurate data for assessing shifts in

110 bacterial and micro/meio-eukaryotic communities. We also evaluated the effects of two different

111 trimming methodologies: one using a minimum read abundance threshold per taxon (i.e.,

112 'trimmed by singletons'), and one removing all taxa not simultaneously recovered from both

113 eDNA and eRNA products of the same environmental sample (i.e., 'trimmed by shared OTUs').

114 We hypothesized that; (1) bacterial and eukaryotic datasets with taxa trimmed by shared eDNA

115 and eRNA products would yield more significant community shifts in response to the effect of

116 the production platform from the removal of most irrelevant OTUs, and 2) that the use of eRNA-

117 derived bacterial and eukaryotic taxa would show stronger correlations with environmental

118 variables and macro-faunal communities than eDNA-derived taxa, as it mostly depicts the living

119 community.

120 


\section{2. Material and Methods}

\section{2.1. Field sampling}

123 The study area was located $80 \mathrm{~km}$ off the west coast of New Zealand's North Island in

124 the South Taranaki Bight (latitude: $-39.97295^{\circ}$, longitude: $173.2997^{\circ}$; Figure 1A). In this region,

125 the seabed has a fairly uniform muddy texture with water depth ranging between 100 to $125 \mathrm{~m}$.

126 Biological samples (bacterial, micro-eukaryotic and macro-faunal assemblages) and physico-

127 chemical data were collected along transects radiating to the East-West axis of one Floating

128 Production Storage and Offloading (FPSO) platform. The FPSO has been in activity since 2009

129 and has been processing and storing the well fluids coming from a wellhead platform (WHP)

130 situated $1.3 \mathrm{~km}$ North-West. The main discharges from the FPSO are production waters

131 (formation water and additives) and operational discharges (sewage, deck drainage, etc.;

132 McConnell et al., 2014). The FPSO site is characterized by a prevalence of anthropogenic debris

133 (e.g. paint chips, construction material, etc.) and coarser, hard-packed and dark-coloured

134 sediments in its vicinity $(\leq 250 \mathrm{~m})$, possibly indicating lower oxygenation level, elevated barium

135 concentrations radiating away from the production water and drilling discharge point, and

136 differences in the structure of macro-faunal assemblages on the east transect, between the near

137 field station $(\leq 250 \mathrm{~m})$ and far-field stations ( $>250 \mathrm{~m}$; Johnston, Elvines \& Newton, 2015).

138 The sampling methodology was based on the Offshore Taranaki Environmental

139 Monitoring Protocol (OTEMP; Johnston et al., 2014), which consisted of a distance-graded

140 sample station allocation. Sampling stations were overlaid along the major flow axis with the

141 FPSO located at the center (Figure 1B). The East-West axis constitutes the main trajectory along

142 which the deposition of drilling mud and cuttings occurs, with the strongest currents usually

143 flowing in a westward direction at a mean speed of $0.12 \mathrm{~ms}^{-1}$ (McConnell et al., 2014). Six

144 stations were sampled at approximately 250, 500 and $1000 \mathrm{~m}$ from the FPSO. 
146 the $15^{\text {th }}$ and $16^{\text {th }}$ of March 2015 (Table S1). Samples were collected with a modified stainless

147 steel double van Veen grab as described in Laroche et al. (2016). To avoid creating a bow wake

148 effect on the seafloor, the grab sampler was deployed at a constant rate of $0.3 \mathrm{~m} / \mathrm{s}$. Recovery of

149 the sampler was similarly processed to avoid displacing superficial sediments. Once retrieved,

150 the top sediment layer was inspected and samples only taken when the surface was undisturbed.

151 The full content of the first grab compartment was sieved $(500 \mu \mathrm{m})$ and preserved in $70 \%$

152 ethanol for analysis of macro-faunal communities. Subsamples (2 g) of undisturbed surface

153 sediment (approximately $1 \mathrm{~cm}$ depth) were collected from the second grab for eDNA/eRNA

154 metabarcoding. These samples were placed in Life Guard ${ }^{\mathrm{TM}}$ Soil Preservation Solution (5 mL;

155 MoBio, USA) to preserve microbial and environmental RNA in stasis, using disposable gloves

156 and spatulas. Samples were stored on ice during transportation to the laboratory and kept frozen

$157\left(-20^{\circ} \mathrm{C}\right)$ until further processing. The second grab compartment was subsampled for

158 physicochemical analyses of sediment as described in Johnston, Elvines \& Newton (2015).

\subsection{Laboratory analyses}

160 2.2.1. Sample processing

161 The macro-fauna was morphologically identified to the lowest practical taxonomic level

162 and enumerated at the Cawthron Institute (Nelson, New Zealand). Sediment texture and chemical

163 analyses were also performed at Cawthron Institute, following the protocol described in Johnston

164 et al. (2014). A summary of all analytical methods used and their detection limit for

165 characterizing the physicochemical parameters of sediment is provided in supplementary

166 information (Table S2). 

Isolation Kit and the DNA Elution Accessory Kit (MoBio, USA). Prior to cDNA synthesis, RNA samples were given two consecutive DNase treatments as described in Langlet et al. (2013). To verify the complete elimination of trace DNA, Polymerase Chain Reaction (PCR) amplifications of 50 cycles were performed using the bacterial forward primers S-D-Bact-0341-b-S-17: 5'-CCT ACG GGN GGC WGC AG-3' and reverse primers S-D-Bact-0785-a-A-21: 5'- GAC TAC HVG GGT ATC TAA TCC-3' (Klindworth et al., 2013), and the eukaryotic forward primers Uni18SF: 5'-AGG GCA AKY CTG GTG CCA GC-3' and reverse primers Uni18SR: 5'-GRC GGT ATC TRA TCG YCT T-3' (Zhan et al., 2013). These primers produce amplicons of ca. $450 \mathrm{bp}$ and were chosen for their ability to capture most bacterial and eukaryotic (especially protist) phyla.

177 Complementary DNA (cDNA) synthesis from single-stranded RNA was performed using

178 random hexamer primers and the SuperScript ${ }^{\circledR}$ III reverse transcriptase (Thermo Fisher

179 Scientific Inc., Massachusetts, USA), all according to the manufacturer's manual. rRNA (V3 and V4 region) and total eukaryote 18S rRNA (V4 region) were amplified using PCR with the same primers descripted above. The primers were modified to include Illumina ${ }^{\mathrm{TM}}$ overhang adaptors forward: 5'-TCG TCG GCA GCG TCA GAT GTG TAT AAG AGA CAG-3' and reverse: 5'- GTC TCG TGG GCT CGG AGA TGT GTA TAA GAG ACA G-3', as PCR Master Mix (Life Technologies), $8 \mu \mathrm{L}$ of double-distilled water $\left(\mathrm{ddH}_{2} \mathrm{O}\right), 1 \mu \mathrm{L}$ of each primer $(10 \mu \mathrm{M}), 5 \mu \mathrm{L}$ of GC enhancer to increase yield, $2 \mu \mathrm{L}(1 \mu \mathrm{g} / \mu \mathrm{L})$ of Bovine Serum 
$190 \mu \mathrm{L})$. Reaction cycling conditions were: $94^{\circ} \mathrm{C}$ for $3 \mathrm{~min}$, followed by 30 cycles of $94^{\circ} \mathrm{C}$ for $30 \mathrm{~s}$,

$19152^{\circ} \mathrm{C}$ for $30 \mathrm{~s}, 72^{\circ} \mathrm{C}$ for $1 \mathrm{~min}$, with a final extension step at $72{ }^{\circ} \mathrm{C}$ for $5 \mathrm{~min}$. For eukaryotes, PCR

192 reactions were performed in a reaction mixture containing $25 \mu \mathrm{L}$ of AmpliTaq Gold® 360 PCR

193 Master Mix (Life Technologies), $13 \mu \mathrm{L}$ of $\mathrm{ddH}_{2} \mathrm{O}, 1 \mu \mathrm{L}$ of each primer $(10 \mu \mathrm{M}), 7 \mu \mathrm{L}$ of $\mathrm{GC}$

194 enhancer, $3 \mu \mathrm{L}$ of BSA, and $3 \mu \mathrm{L}$ of template eDNA (1.58 to $3.96 \mathrm{ng} / \mu \mathrm{L})$ or $1.5 \mu \mathrm{L}$ of eRNA

$195(10 \mathrm{ng} / \mu \mathrm{L})$. Reaction cycling conditions were: $95^{\circ} \mathrm{C}$ for $5 \mathrm{~min}$, followed by 35 cycles of $94^{\circ} \mathrm{C}$

196 for $30 \mathrm{~s}, 54^{\circ} \mathrm{C}$ for $30 \mathrm{~s}, 72^{\circ} \mathrm{C}$ for $45 \mathrm{~s}$, with a final extension step at $72^{\circ} \mathrm{C}$ for $7 \mathrm{~min}$. All PCR

197 amplifications included negative controls (no template samples) to ensure amplification of

198 uncontaminated products. Amplicons were purified with Agencourt ${ }^{\circledR}$ AMPure ${ }^{\circledR}$ XP PCR

199 Purification beads (Beverly, Massachusetts, USA) following the manufacturers' instructions.

200 Purified products were quantified using a Qubit ${ }^{\circledR}$ Fluorometer (Life Technologies, Carlsbad,

201 California, USA), and diluted to $3 \mathrm{ng} / \mu \mathrm{L}$ using $\mathrm{ddH}_{2} \mathrm{O}$. Each product was individually indexed

202 using the Nextera ${ }^{\mathrm{TM}}$ DNA library Prep Kit (Illumina), pooled into two libraries (eDNA and 203 eRNA) and sequenced on a MiSeq ${ }^{\mathrm{TM}}$ Illumina platform using a $2 \times 250$ base pair paired-end

204 protocol at the New Zealand Genomics Ltd. (Auckland, New Zealand). A sample containing 20

$205 \mu \mathrm{L}$ of $\mathrm{ddH}_{2} \mathrm{O}$ was used as a negative control for the multiplexing and sequencing steps in each

206 library. Internal sequencing quality control was also ensured by including DNA samples from

207 three previously isolated marine species (i.e., Sabella spallanzanii, Ciona savignyi and Perna

208 perna (Pochon et al. 2013) that were PCR amplified, purified and quantified following the

209 eukaryote DNA protocol described above. These species were pooled at equimolar concentration

210 and included in each sequencing run. The raw sequencing reads are publicly available in the

211 Sequence Read Archive (SRA) under the accession numbers SRR5215546 to SRR5215617. 
212 Additionally, the filtered sequence dataset is available for download on FigShare at the following

213 link: https://figshare.com/articles/Laroche_et_al_2017_Maari/4600174

214 2.2.3. Bioinformatics analysis

215 Using VSEARCH (Rognes et al., 2016), raw FASTQ reads were truncated from the first

216 base where the Q score dropped below 3, and paired-end assembled with a minimum merge

217 length of 200 reads. Merged reads with more than one base-pair error were discarded and the

218 remaining demultiplexed. The bacteria and eukaryote data were concatenated into one file each

219 (bacteria.fasta, eukaryote.fasta) and dereplicated. Unique sequences of bacterial 16S rRNA were

220 compared to the Ribosomal Database Project (RDP; Cole et al., 2014), and unique sequences of

221 eukaryotic 18S rRNA mapped against the Protist Ribosomal 2 database (PR2; Guillou et al.,

222 2013) for chimera detection. Prior to clustering reads into operational taxonomic units (OTUs)

223 with a 97\% similarity, representative sequences were sorted by abundance so that the most

224 important sequences would be at the start of the file and used as seed for the clustering.

225 Taxonomy was assigned using the QIIME package (Caporaso et al., 2010) and the uclust

226 assigner. By default, the program performs a search to record reference sequences matching a

227 minimum of $90 \%$ of each query entry (OTU), and assign the most specific taxonomic label

228 according to a minimum consensus fraction ( 0.51 by default) of the matches. This approach

229 enabled taxonomic assignment of poorly represented taxa in reference databases. Operational

230 taxonomic units with no match were discarded.

231 Rarefaction curves were generated in QIIME using the Chaol and Shannon metrics

232 (Figure S1). This analysis demonstrated that approximately 2,500 reads per sample for both

233 bacteria and eukaryote datasets were required to adequately capture the diversity within each

234 sample. Samples with $<2,500$ reads ( 1 for bacterial eDNA and 1 for eukaryote eDNA and eRNA 
235 samples) were discarded from subsequent analyses (Table S1). To enable robust comparisons

236 between eDNA and eRNA datasets, an equivalent number of samples were kept per station for

237 each type of molecule, resulting in the additional removal of one bacterial eRNA sample (Table

238 S1).

239 For the remaining data manipulation and analysis, three datasets were used for both

240 bacteria and eukaryotes; (1) containing all OTUs (referred to as 'untrimmed'), (2) only

241 containing OTUs with at least two unique sequence reads present within the whole dataset

242 (referred to as 'trimmed by singletons'), and (3) including only the OTUs found in both DNA

243 and RNA compartments from each sample (referred to as 'trimmed by shared OTUs').

244 2.3. Data analysis and statistics

245 Samples were grouped into near-field ( $\leq 250 \mathrm{~m}$ of the FPSO) and far-field $(>250 \mathrm{~m})$

246 stations from each side of the FPSO. To visualize the effect of trimming by singletons and by

247 shared OTUs, the number of reads and OTUs in each dataset were compared. The OTUs

248 removed from the shared OTUs trimming method were further investigated by displaying bar

249 plots of the relative abundance of the ten most important classes for bacteria and phyla for

250 eukaryotes using the R package 'phyloseq' (McMurdie and Holmes, 2013), and by performing a

251 Mann-Whitney $U$ test with 999 permutations on these taxa to detect significant abundance

252 differences between the near- and far-field stations.

253 Prior to further analysis, read counts were normalized among samples. The large

254 discrepancy in read abundance among samples (up to 5 times) prevented the use of typical

255 normalization techniques such as variance stabilization (R package DESeq2; Love, Anders \&

256 Huber, 2014) or cumulative sum scaling (R package metagenomeSeq; Paulson et al., 2013), and

257 a subsampling-based normalization strategy was employed following the method described in 
258 Aguirre de Carcer et al. (2011). Briefly, multiple rarefactions (100 times) based on the median

259 number of reads among all samples were performed, followed by a rarefaction to the lowest

260 number of reads among all samples. Since a large number of singletons may represent PCR or

261 sequencing artefacts (Majaneva et al., 2015; Brown et al., 2015), these were removed prior to

262 statistical analysis.

263 Using normalized data kept after the two different trimming techniques, observed OTUs

264 and the Shannon diversity index were analyzed for each sample. To test for the effect of the

265 FPSO on the bacterial and eukaryotic alpha-diversity of eDNA/eRNA datasets, a nonparametric

266 t-test using 999 permutations was performed between near and far-field stations. To determine

267 whether the eDNA or eRNA dataset contained the most abundant number of OTUs significantly

268 affected by the FPSO activity, a non-parametric t-test with 999 permutations was conducted on

269 OTUs present in at least three samples, between the near- and far-field station groups.

270 Differences in beta-diversity between and within the near- and far-field stations were tested

271 using an Adonis and PERMDISP tests with 999 permutations on Bray-Curtis distance matrices,

272 using square root transformed data. Non-metric multidimensional scaling plots were obtained

273 using the R package 'phyloseq' (McMurdie \& Holmes, 2013). Correlations between bacterial

274 and eukaryotic eDNA/eRNA OTUs and macro-fauna beta-diversities, and with environmental

275 variables were investigated using a Mantel test based on 999 permutations. The tests were

276 performed on square root transformed data using Bray-Curtis distance matrices for biological

277 data, and an Euclidean distance matrix for environmental data. For the later, only variables

278 showing a significant change between the near-field and far-field stations were used (i.e., barium

279 and AFDW; Johnston et al., 2015), and square root transformed prior to the distance matrix 
280 computation. Except for the bar plots and nMDS, all analyses mentioned above were computed

281 using the QIIME pipeline (Caporaso et al., 2010).

\section{3. Results}

\section{3.1. High-throughput sequencing output}

284 Each of the 18 sediment samples collected in this study were co-extracted for both eDNA 285 and eRNA and PCR-amplified for total bacteria and eukaryotes, resulting in the total production 286 of 72 PCR-amplicons (36 for bacteria and 36 for eukaryotes; Tables S1). The no template PCR

287 controls remained negative in all PCR trials. All 72 amplicons were sequenced on an Illumina ${ }^{\mathrm{TM}}$ 288 MiSeq instrument, yielding a total of 2,134,982 and 1,040,624 eDNA and eRNA sequence reads, 289 respectively. The water blank negative controls $\left(\mathrm{ddH}_{2} \mathrm{O}\right.$ sample) contained a total of 524 raw 290 reads (130 for bacteria and 494 for eukaryotes) in the eDNA library, and 405 raw reads (234 for 291 bacteria and 171 for eukaryotes) in the eRNA library, of which none remained after quality 292 filtering. The positive controls were assigned at $99.9 \%$ to the three target taxa. After removal of 293 unmatched and low-quality reads, a total of 883,155 reads and 33,995 OTUs were obtained for 294 bacteria (eDNA: 25,100; eRNA: 22,211), and a total of 768,374 reads and 11,854 OTUs (eDNA: 295 7,045; eRNA: 8,059) for eukaryotes. Removal of samples with insufficient diversity coverage 296 led to the removal of one bacterial eDNA and eRNA sample (FE250b), and one eukaryotic 297 eDNA (FE250b) and eRNA (FE250c) sample (Table S1).

\subsection{Effect of trimming methodologies}

For the bacterial dataset, the removal of singletons (i.e., OTUs present only once across

300 the entire eDNA/eRNA datasets) resulted in a $1 \%$ loss of total reads and $37 \%$ loss of OTUs

301 (Table 1). Similar results were observed for eukaryotes where $0.4 \%$ of reads and $29 \%$ of OTUs

302 were removed. Trimming OTUs that did not occur in both eDNA/eRNA amplicons of the same 
303 sample resulted in a total reduction in OTUs of $78 \%$, and total read reduction of $27 \%$. For

304 eukaryote data, trimming by shared OTUs had a larger impact on OTU and read removal,

305 discarding an additional $51 \%$ of OTUs for a total reduction of $80 \%$, and reducing the total read

306 abundance by $28 \%$. The mean number of reads (counts) per removed OTUs was of 3.1 and 8.6

307 for bacteria and eukaryotes respectively.

308 Figure 2 summarizes the total number of bacterial and eukaryotic OTUs that were either

309 shared between both eDNA and eRNA data of the whole datasets, shared between eDNA and

310 eRNA from the same sample, or those that were restricted to one dataset type following the two

311 distinct data treatments. The percentage of shared OTUs between the eDNA and eRNA bacterial

312 datasets increased from $22 \%$ to $36 \%$ after singleton removal (Table 1 ). For eukaryotes, removing

313 singletons improved the percentage of shared OTUs in the eDNA and eRNA datasets from 20 to

$31428 \%$. The percentage of unshared OTUs were highest in the eDNA dataset for bacteria, while in

315 the eukaryotic dataset most of the unshared OTUs were in the eRNA.

316 After trimming by singletons, the bacterial datasets (kept OTUs) were mainly composed

317 of Gammaproteobacteria (eDNA 57.2\%, eRNA 77.9\%), Deltaproteobacteria (eDNA 12\%, eRNA

318 8\%) and Alphaproteobacteria (eDNA 8.5\%, eRNA 4.8\%; Table S3). Metazoa composed the

319 large majority of eukaryote OTUs (eDNA 84.6\%, eRNA 85.5\%), followed by Dinophyta (6.2\%)

320 and Radiolaria (2.8\%) in eDNA, and by Cercozoa (7.4\%) and Cilliophora (3.5\%) in eRNA

321 (Table S3).

Trimming by shared OTUs slightly changed the relative abundance of each taxa of the

323 kept data, but did not change their decreasing order (Table S4). The majority of bacterial OTUs

324 removed from trimming by shared OTUs were Proteobacteria belonging to Deltaproteobacteria 
325 (eDNA 19\%, eRNA 30.4\%) and Gammaproteobacteria (eDNA 22.7\%, eRNA 28.2\%; Figure 3,

326 Table S3). In eDNA, the taxonomic groups Planctomycetia (9.6\%), Alphaproteobacteria (7.1\%)

327 and Verrucomicrobiae (5.7\%) also accounted for a substantial part of the trimmed OTUs. Most

328 of the eukaryote OTUs removed were Metazoa (eDNA 60.5\%, eRNA 42.8\%), followed by

329 Dinophyta (13.9\%) and Apicomplexa (10.1\%) in eDNA, and by Ciliophora (19.7\%) and

330 Cercozoa (19.7\%) in eRNA (Table S3). Among all these groups, only eDNA Verrucomicrobiae

$331(\mathrm{~T}$-stat $=2.39, \mathrm{P}$-value $=0.03 ;$ Table S6) and eRNA Planctomycetia $(\mathrm{T}$-stat $=-2.78, \mathrm{P}$-value $=$

332 0.03; Table S6) expressed a significant relation with distance from the platform, both being more

333 abundant in the near-field stations.

334 3.3. Comparison of bacteria and eukaryotes eDNA and eRNA datasets

335 3.3.1 Alpha-diversity

336 Non-parametric t-tests on alpha-diversity metrics of the eDNA dataset trimmed by

337 singletons (kept OTUs) showed that there was no significant difference between the near- and

338 far-field stations for bacteria or eukaryotes (Table 2). The same was observed with the equivalent

339 eRNA bacterial data, and eRNA eukaryote data. When using the 'trimmed by shared OTUs'

340 method, significant differences between the near- and far-field stations for the observed OTUs

341 metric were observed with bacterial eDNA ( $\mathrm{p}$-value $=<0.01)$ and with eukaryotic eDNA (p-

342 value $=0.04)$, and for the Shannon index with bacterial eDNA ( $\mathrm{p}$-value $=<0.01)$.

343 3.3.2. Significant OTUs, beta-diversity and correlation to environmental variables

344 The non-parametric t-test analysis identified OTUs significantly associated with the

345 near- or far-field station groups, by comparing their frequencies across samples groups. For both

346 taxa, these OTUs were more abundant in eDNA than eRNA (Table S7). Trimming by shared 
347 OTUs reduced the number of significant OTUs by 52 (eDNA) and 26 (eRNA) in bacteria, and by

34899 (eDNA) and 86 (eRNA) in eukaryotes (Table S7).

349 Non-metric multidimensional scaling plots (Figure 4) demonstrated that near- and far-

350 field stations tended to cluster separately for both taxa. Our distance-based test for homogeneity

351 of multivariate dispersion (Permdisp) indicated that communities within the near- and far-field

352 stations groups were homogeneous in all datasets (Table S8). However, significant variance

353 between replicates were observed for bacterial eDNA $(p$-value $=0.03)$ and eRNA ( $p$-value $=$

$3540.04)$ data of the trimmed by singleton dataset, and for bacterial eDNA ( $p$-value $=0.03$ ) of the

355 trimmed by shared OTUs dataset (Table S9).

356 When comparing the correlation of bacterial eDNA/eRNA beta-diversity matrices

357 between near- and far-field stations, eDNA provided stronger correlations and/or lower p-values

358 (Table 3). This trend was also observed with correlations to the distance matrices of macro-fauna

359 and environmental variables (Table 3). For eukaryotes however, this tendency was not always

360 observed as eRNA showed slightly stronger correlations and more significant results between the

361 near- and far-field stations, but weaker correlations with macro-faunal assemblages and with

362 environmental variables. Overall, correlations between eDNA and eRNA beta-diversities were

363 stronger for the trimmed by shared OTUs datasets, and only significantly positive among the

364 eukaryote datasets (Table 3). For bacteria, the trimmed singletons datasets demonstrated

365 particularly weak correspondence $(r=-0.05$; $\mathrm{p}$-value $=0.8)$ between eDNA and eRNA. 


\section{4. Discussion}

The aims of this study were to assess whether high throughput sequencing (HTS) data

obtained from eDNA and/or eRNA provide the best proxy for evaluating environmental impacts and to explore how data processing steps may influence these results. We focused on benthic habitats at bathysmal depth, within close proximity to an offshore oil production platform.

\subsection{High-throughput sequencing outputs}

In our datasets, the number of bacterial eDNA OTUs (Table 1) was slightly higher than eRNA. Environmental DNA is more resistant to posthumous degradation, and may persist several years in marine sediment (Dell'Anno, 2005). Analysis of the eukaryote data showed a differing pattern with more eRNA OTUs compared to eDNA. The proportion of OTUs found only in eDNA or eRNA datasets varies substantially among previous investigations. For example, Dowle et al. (2015) found more bacterial eRNA than eDNA OTUs in their datasets, and $\mathrm{Hu}$ et al. (2016) and Laroche et al. (2016) obtained more protist eRNA than eDNA OTUs, while Pawlowski et al. (2014) and Pochon et al. (2015) detected more protist eDNA than eRNA

381 OTUs. It is possible that many of the unshared eRNA OTUs represent PCR artefacts, especially

382 from the reverse transcription of RNA to cDNA, and sequencing errors (Egge et al., 2013;

383 Ficetola et al., 2015). The RNA conversion to complementary DNA (cDNA) requires the use of 384 a reverse transcriptase which, as opposed to DNA polymerase, lack proof reading activity, creating point mutations in some of the cDNA sequences (Svarovskaia et al., 2003; Houseley \&

386 Tollervey, 2010). Reverse transcriptase also frequently 'jump' from one template to another

387 during the transcription process. Known as template-switching, this action can lead to two 388 different outcomes: 1) production of chimeric cDNA sequences from intermolecular template switching, and 2) creation of shortened isoform sequences from intramolecular template 
390 switching (Cocquet et al., 2006). The latter being more problematic as it is unlikely that this can

391 be detected during bioinformatics analysis. Finally, the use of random hexamer primers for

392 cDNA synthesis is known to introduce nucleotide bias at the beginning of the 5 '-end of sequence

393 reads (Hansen, Brenner \& Dudoit, 2010). Because the pre-mentioned artefacts are subsequently

394 augmented by the gene-specific PCR amplification, they would become integrated into the HTS

395 library and potentially not be removed during bioinformatics filtering processes. The incertitude

396 linked to potential intrinsic error rates from reverse transcription limits accurate comparison of

397 eDNA and eRNA datasets. The use of a RNA control (e.g., synthetic oligomers) and technical

398 (PCR) replicates could help identifying these artefacts and improve concordance between the

399 eDNA and eRNA profiles. Trimming OTUs by their shared presence in eDNA and eRNA

400 represents an alternative way of discarding reverse transcription artefacts.

401 In this study, bacterial and eukaryote RNAs were treated using the same protocol during

402 the reverse transcription process. However, eukaryote cDNA required more PCR cycles (35

403 instead of 30 for bacteria) to amplify sufficient material, potentially producing more false

404 transcripts and explaining the differences observed between the two taxonomic groups (Figure

405 2). Another possibility could be the presence of rare but highly active eukaryote OTUs which

406 were not detected in the eDNA dataset because of their low abundance. Particularly high activity

407 and growth from some of the rare biota has been previously reported for both bacteria (Campbell

408 et al., 2011; Hugoni et al., 2013) and microbial eukaryotes (Logares et al., 2014).

\subsection{Effect of trimming methodologies}

410 Trimming OTUs not simultaneously present in both eDNA and eRNA assemblages

411 from the same samples resulted in twice as many OTUs being removed compared to discarding

412 singletons only, and removed 27-fold (bacteria) and 70-fold (eukaryotes) more reads. However, 
413 further investigation suggested that it mostly resulted in the removal of legacy DNA, DNA

414 deposited from the water column, and technical artefacts. This improved the sensitivity and

415 accuracy of our environmental metabarcoding analysis (Tables 2 and 3) and enabled the

416 detection of differences between the effects of the production platform on near- and far-field

417 stations. In particular, it allowed us to disclose the divergence between beta-diversities of near-

418 field and far-field stations, and improved the correlations between the beta-diversity and

419 environmental variables distance matrices (Table 3).

Removal of single sequence detection (singletons) to reduce PCR and sequencing

421

422

423

424

425

426

427

428

430

431

432

433

434

435 Monfort, Guillera-Arroita \& Tingley, 2016). 
437 artefacts. This approach allows for an informed elimination of artefactual OTUs while also

438 discarding data from legacy DNA. However, trimming data by shared OTUs between co-

439 extracted eDNA and eRNA material is particularly stringent and may result in the removal of a

440 substantial number of false negatives. As an alternative, biological or technical replicates could

441 be used in parallel to discriminate genuine and false detections. For examples, biological

442 replicates could be pooled together prior to trimming by shared OTUs, and subsequently

443 demultiplexed to allow evaluation of within station variability. It is worth mentioning that the

444 application of unsupervised oligotyping methodologies such as the one proposed by Eren et al.

445 (2015), could further improve sensitivity of environmental metabarcoding monitoring studies by

446 discriminating closely related taxa, and will likely gain in popularity as tools are being

447 developed.

\subsection{Operational taxonomic units not shared between both eDNA and eRNA datasets}

Among the bacterial datasets, most of the removed OTUs (i.e., those not simultaneously

found in both eDNA and eRNA) belonged to Gammaproteobacteria and Deltaproteobacteria,

which also constituted the main classes of the singleton trimmed dataset. This suggests that most

452 OTUs removed by the shared OTUs trimming method may have arisen from PCR or sequencing

errors originating from highly abundant unique sequences which subsequently associated to

these classes (Huse et al., 2010; Quince et al., 2011). Another possibility could be that these

abundant taxa have more polymorphisms. In such cases, removal of these rare variants would

produce false negatives. Nonetheless, their small contribution to weighted (abundance-based)

analyses, as demonstrated in the present study, would unlikely affect results. 
459 Metazoa, Dinophyta and Apicomplexa in eDNA, and Ciliophora and Cercozoa in eRNA (Figure

4603 , Table S5). These phyla are also the most abundant in the datasets trimmed by singletons

461 (Table S3). None of these showed a significant correlation with distance from the platform

462 (Table S6). Just as for bacteria, the important abundance of these phyla in the discarded

463 eDNA/eRNA OTUs is likely a consequence of PCR/sequencing errors originating from the most

464 abundant unique sequences. This is further supported by the fact that the decreasing order of

465 relative abundance of the most important taxa is the same between the datasets of kept OTUs and

466 removed OTUs resulting from the shared OTUs trimming.

\subsection{Comparison between eDNA and eRNA}

When performing alpha-diversity on the trimmed by singletons datasets, differences were observed between eDNA and eRNA, with the latter mainly showing stronger dissimilarities between the near-field and far-field stations. This is not surprising as eDNA can contain genetic information of past and current communities, inflating alpha-diversity and potentially masking the present effects of the platform (Corinaldesi, Danovaro \& Dell'Anno, 2005; Dell'Anno, 2005).

Beta-diversity analysis of the singleton trimmed data showed more significant differences between the near- and far-field stations with bacterial eDNA, while only small differences could be observed between eukaryote eDNA and eRNA. Relationships between micro-faunal and the macro-faunal assemblages and environmental variables were also much stronger than with eDNA. This suggest that while eRNA may represent a better proxy for assessing ongoing anthropogenic effects on species diversity, eDNA seems more reliable for assessing effects on community composition. This is especially true for bacteria where no correlation could be observed between eRNA data and macro-fauna, or with environmental variables (Table 2). These 
482 results contrast with other similar studies (Pawlowski et al., 2014; Visco et al., 2015; Dowle et

483 al., 2015; Pochon et al., 2015; Laroche et al., 2016; Hu et al., 2016) where eRNA always out-

484 performed eDNA when correlating micro- and macro-faunal assemblages, or when correlating

485 these assemblages with environmental conditions. Nonetheless, these studies used a less

486 stringent OTU filtering method prior to statistical analyses, removing only those not present in

487 both eDNA/eRNA compartment of their whole dataset. Consequently, an important number of

488 OTUs from legacy DNA may have subsisted in their datasets, decreasing the observed sensitivity

489 to local conditions of the eDNA-derived communities. Also, the relationship between eRNA

490 concentrations and activity does not always align. Differences such as life history, life strategies

491 and non-growth activity between organisms play an important role in RNA production

492 (Blazewicz et al., 2013). Ribosomal eRNA such as those targeted in this study (16S and 18S) can

493 have a much wider range of expression (over 3 orders of magnitude) than ribosomal eDNA (ca. 1

494 order of magnitude; Fegatella et al., 1998). Fluctuation in DNA copy number between taxa and

495 in RNA expression may account for the strong variability observed among the bacterial eDNA

496 and eRNA replicates (Table S8). This variability increases confidence intervals, reduces

497 statistical power, and increases the risk of not identifying impacts (Type II errors; Underwood, 498 1993).

For accurate assessment of false positive and negative OTU occurrences, and sufficient 500 evaluation of within station biological variability, optimal number of replicate samples is often a

501 compromise between available funds and time, and the desired level of precision and sensitivity.

502 Parameters such as targeted taxa, the choice of primer set and the sequencing depth of HTS

503 libraries all play an important part in taxa detection probability, which in turn, dictates the

504 optimal replication level (Ficetola et al., 2015). In studies assessing whole communities, the 
505 number of replicates should ideally depend on the taxa with the lowest detection probability.

506 Ficetola et al. (2015) give an example where a taxon with a 0.5 probability would normally

507 require a minimum of eight replicates to ensure detection. For routine monitoring surveys of

508 benthic communities however, where the non-detection of rare biota is unlikely to change the

509 outcomes of studies, the replication level does not have to be as high. In such cases, the number

510 of replicates may be more influenced by the level of heterogeneity found within stations.

511 An important consideration when using single-time sampling designs over a

512 contamination gradient is that it assumes that natural factors equally influence communities

513 among samples (Wiens \& Parker, 1995). This bias can be particularly problematic with small

514 and short-lived organisms like bacteria. In low impacted environments, such as those explored in

515 this study, biological interactions may supplant the effect of environmental factors, increasing

516 spatial variability (Schafer, 2000), and creating stronger discrepancies among biological

517 replicates. In this situation, collecting more biological replicates per stations should be favoured

518 over technical replicates, in order to mitigate the effect of micro-patchiness.

\section{5. Conclusion}

520 The resolution, sensitivity and accuracy of metabarcoding techniques used to measure

521 anthropogenic impact on microbial communities can be substantially affected by whether eDNA

522 or eRNA is used and by the choice of pre-analysis data treatment method. Disparities in results

523 may also occur between eukaryote and bacterial eDNA/eRNA, that should be considered before

524 analyzing environmental metabarcoding monitoring data. Our study showed that when OTUs are

525 solely trimmed by singletons, eRNA represents a better proxy for alpha-diversity as it is more

526 likely to contain living biota only. Nonetheless, read abundance information from eDNA data

527 was, for bacteria, superior to its counterpart for assessing environmental impacts on beta- 
528 diversity. In weakly impacted environments, this may be even more important as eRNA

529 concentration can be highly dependent and sensitive to temporal interactions from restricted local

530 conditions that can be unrelated to the anthropogenic activity. For this reason, read abundance

531 information from bacterial eDNA seems more suited for assessing the effect of oil and gas

532 drilling and production operations, where closest monitored stations are located far from the

533 pollution source (ca. $250 \mathrm{~m}$ ). Overall, trimming OTUs not shared in both DNA and RNA

534 compartments of each sample improved the sensitivity and accuracy of our results. While this is

535 true for our study site, further investigations will be needed to evaluate the extent of these

536 findings.

537

538

539

540 


\section{Acknowledgements}

542

543 We thank Olivia Johnston and Deanna Elvines (Cawthron) for sample collection, OMV New

544 Zealand Limited for providing access to samples and corresponding environmental data.

545

546

547

548

549

550

551

552

553

554

555

556

557

558

559

560

561

562

563

564

565

566

567

568

569

570

571 
572

573

574

575

576

577

578

579

580

581

582

583

584

585

586

587

588

589

590

591

592

593

594

595

596

597

598

599

600

601

602

603

604

605

606

607

608

609

610

611

612

613

614

615

616

617

\section{References}

Abad D., Albaina A., Aguirre M., Laza-Martínez A., Uriarte I., Iriarte A., Villate F., Estonba A. 2016. Is metabarcoding suitable for estuarine plankton monitoring? A comparative study with microscopy. Marine Biology 163:149. DOI: 10.1007/s00227-016-2920-0.

Aguirre de Carcer D., Denman SE., McSweeney C., Morrison M. 2011. Evaluation of subsampling-based normalization strategies for tagged high-throughput sequencing data sets from gut microbiomes. Applied and Environmental Microbiology 77:8795-8798. DOI: 10.1128/AEM.05491-11.

Bálint M., Bahram M., Eren AM., Faust K., Fuhrman JA., Lindahl B., O’Hara RB., Öpik M., Sogin ML., Unterseher M., Tedersoo L. 2016. Millions of reads, thousands of taxa: microbial community structure and associations analyzed via marker genes. FEMS Microbiology Reviews 6:fuw017. DOI: 10.1093/femsre/fuw017.

Beng KC., Tomlinson KW., Shen XH., Surget-Groba Y., Hughes AC., Corlett RT., Slik JWF. 2016. The utility of DNA metabarcoding for studying the response of arthropod diversity and composition to land-use change in the tropics. Scientific reports 6:24965. DOI: $10.1038 /$ srep24965.

Blazewicz SJ., Barnard RL., Daly RA., Firestone MK. 2013. Evaluating rRNA as an indicator of microbial activity in environmental communities: limitations and uses. The ISME Journal 7:2061-2068. DOI: 10.1038/ismej.2013.102.

Bohmann K., Evans A., Gilbert MTP., Carvalho GR., Creer S., Knapp M., Yu DW., de Bruyn M. 2014. Environmental DNA for wildlife biology and biodiversity monitoring. Trends in Ecology and Evolution 29:358-367. DOI: 10.1016/j.tree.2014.04.003.

Bourlat SJ., Borja A., Gilbert J., Taylor MI., Davies N., Weisberg SB., Griffith JF., Lettieri T., Field D., Benzie J., Glöckner FO., Rodríguez-Ezpeleta N., Faith DP., Bean TP., Obst M. 2013. Genomics in marine monitoring: New opportunities for assessing marine health status. Marine Pollution Bulletin 74:19-31. DOI: 10.1016/j.marpolbul.2013.05.042.

Brown SP., Veach AM., Rigdon-Huss AR., Grond K., Lickteig SK., Lothamer K., Oliver AK., Jumpponen A. 2015. Scraping the bottom of the barrel: are rare high throughput sequences artifacts? Fungal Ecology 13:221-225. DOI: 10.1016/j.funeco.2014.08.006.

Campbell BJ., Yu L., Heidelberg JF., Kirchman DL. 2011. Activity of abundant and rare bacteria in a coastal ocean. Proceedings of the National Academy of Sciences of the United States of America 108:12776-12781. DOI: 10.1073/pnas.1101405108.

Caporaso JG., Kuczynski J., Stombaugh J., Bittinger K., Bushman FD., Costello EK., Fierer N., Peña AG., Goodrich JK., Gordon JI., Huttley G a., Kelley ST., Knights D., Koenig JE., Ley RE., Lozupone C a., McDonald D., Muegge BD., Pirrung M., Reeder J., Sevinsky JR., Turnbaugh PJ., Walters W a., Widmann J., Yatsunenko T., Zaneveld J., Knight R. 2010. QIIME allows analysis of high-throughput community sequencing data. Nature Methods 7:335-336. DOI: 10.1038/nmeth.f.303.

Carew ME., Pettigrove VJ., Metzeling L., Hoffmann A a. 2013. Environmental monitoring using next generation sequencing: rapid identification of macroinvertebrate bioindicator species. Frontiers in zoology 10:45. DOI: 10.1186/1742-9994-10-45.

Chariton AA., Ho KT., Proestou D., Bik H., Simpson SL., Portis LM., Cantwell MG., Baguley JG., Burgess RM., Pelletier MM., Perron M., Gunsch C., Matthews RA. 2014. A molecularbased approach for examining responses of eukaryotes in microcosms to contaminantspiked estuarine sediments. Environmental Toxicology and Chemistry 33:359-369. DOI: 
618

619

620

621

622

623

624

625

626

627

628

629

630

631

632

633

634

635

636

637

638

639

640

641

642

643

644

645

646

647

648

649

650

651

652

653

654

655

656

657

658

659

660

661

662

663

10.1002/etc. 2450 .

Charvet S., Vincent WF., Lovejoy C. 2014. Effects of light and prey availability on Arctic freshwater protist communities examined by high-throughput DNA and RNA sequencing. FEMS Microbiology Ecology 88:550-564. DOI: 10.1111/1574-6941.12324.

Cocquet J., Chong A., Zhang G., Veitia RA. 2006. Reverse transcriptase template switching and false alternative transcripts. Genomics 88:127-131. DOI: 10.1016/j.ygeno.2005.12.013.

Corinaldesi C., Danovaro R., Dell'Anno A. 2005. Simultaneous recovery of extracellular and intracellular DNA suitable for molecular studies from marine sediments. Applied and Environmental Microbiology 71:46-50. DOI: 10.1128/AEM.71.1.46-50.2005.

Dell'Anno A. 2005. Extracellular DNA plays a key role in deep-sea ecosystem functioning. Science 309:2179-2179. DOI: 10.1126/science.1117475.

Dowle E., Pochon X., Keeley N., Wood SA. 2015. Assessing the effects of salmon farming seabed enrichment using bacterial community diversity and high-throughput sequencing. FEMS Microbiology Ecology 91:fiv089. DOI: 10.1093/femsec/fiv089.

Egge E., Bittner L., Andersen T., Audic S., de Vargas C., Edvardsen B. 2013. 454 pyrosequencing to describe microbial eukaryotic community composition, diversity and relative abundance: a test for marine haptophytes. PLoS ONE 8:e74371. DOI: 10.1371/journal.pone.0074371.

Eren AM., Morrison HG., Lescault PJ., Reveillaud J., Vineis JH., Sogin ML. 2015. Minimum entropy decomposition: unsupervised oligotyping for sensitive partitioning of highthroughput marker gene sequences. Isme J 9:968-979. DOI: 10.1038/ismej.2014.195.

Ficetola GF., Pansu J., Bonin A., Coissac E., Giguet-Covex C., De Barba M., Gielly L., Lopes CM., Boyer F., Pompanon F., Rayé G., Taberlet P. 2015. Replication levels, false presences and the estimation of the presence/absence from eDNA metabarcoding data. Molecular ecology resources 15:543-56. DOI: 10.1111/1755-0998.12338.

Ficetola GF., Taberlet P., Coissac E. 2016. How to limit false positives in environmental DNA and metabarcoding? Molecular Ecology Resources 16:604-607. DOI: 10.1111/17550998.12508.

Gupta RS. 2016. Impact of genomics on the understanding of microbial evolution and classification: The importance of Darwin's views on classification. FEMS Microbiology Reviews 40:520-553. DOI: 10.1093/femsre/fuw011.

Hansen KD., Brenner SE., Dudoit S. 2010. Biases in Illumina transcriptome sequencing caused by random hexamer priming. Nucleic Acids Research 38:1-7. DOI: 10.1093/nar/gkq224.

Houseley J., Tollervey D. 2010. Apparent non-canonical trans-splicing is generated by reverse transcriptase in vitro. PLOS ONE 5. DOI: 10.1371/journal.pone.0012271.

Hu SK., Campbell V., Connell P., Gellene AG., Liu Z., Terrado R., Caron DA. 2016. Protistan diversity and activity inferred from RNA and DNA at a coastal ocean site in the eastern North Pacific. FEMS Microbiology Ecology:fiw050. DOI: 10.1093/femsec/fiw050.

Hugoni M., Taib N., Debroas D., Domaizon I., Jouan Dufournel I., Bronner G., Salter I., Agogué H., Mary I., Galand PE. 2013. Structure of the rare archaeal biosphere and seasonal dynamics of active ecotypes in surface coastal waters. Proceedings of the National Academy of Sciences of the United States of America 110:6004-9. DOI: 10.1073/pnas. 1216863110.

Hunt DE., Lin Y., Church MJ., Karl DM., Tringe SG., Izzo LK., Johnson ZI. 2013. Relationship between abundance and specific activity of bacterioplankton in open ocean surface waters. Applied and Environmental Microbiology 79:177-184. DOI: 10.1128/AEM.02155-12.

Peer] reviewing PDF | (2017:01:15580:1:1:NEW 20 Mar 2017) 
664 Huse SM., Welch DM., Morrison HG., Sogin ML. 2010. Ironing out the wrinkles in the rare

665

666

667

668

669

670

671

672

673

674

675

676

677

678

679

680

681

682

683

684

685

686

687

688

689

690

691

692

693

694

695

696

697

698

699

700

701

702

703

704

705

706

707

708

709

biosphere through improved OTU clustering. Environmental Microbiology 12:1889-1898.

DOI: $10.1111 / \mathrm{j} .1462-2920.2010 .02193 . x$.

Ji Y., Ashton L., Pedley SM., Edwards DP., Tang Y., Nakamura A., Kitching R., Dolman PM., Woodcock P., Edwards F a., Larsen TH., Hsu WW., Benedick S., Hamer KC., Wilcove DS., Bruce C., Wang X., Levi T., Lott M., Emerson BC., Yu DW. 2013. Reliable, verifiable and efficient monitoring of biodiversity via metabarcoding. Ecology Letters 16:1245-1257. DOI: 10.1111 ele.12162.

Johnston O., Barter P., Ellis J., Elvines D. 2014. Recommandations for an offshore Taranaki environmental monitoring protocol: Drilling and production related discharges. Nelson.

Johnston O., Elvines D., Newton M. 2015. Benthic ecological investigation for the maari facilities: annual production and mid-drill assessment, March 2015. Nelson.

Klindworth A., Pruesse E., Schweer T., Peplies J., Quast C., Horn M., Glockner FO. 2013. Evaluation of general 16S ribosomal RNA gene PCR primers for classical and nextgeneration sequencing-based diversity studies. Nucleic Acids Research 41:e1-e1. DOI: 10.1093/nar/gks808.

Kozich JJ., Westcott SL., Baxter NT., Highlander SK., Schloss PD. 2013. Development of a dual-index sequencing strategy and curation pipeline for analyzing amplicon sequence data on the miseq illumina sequencing platform. Applied and Environmental Microbiology 79:5112-5120. DOI: 10.1128/AEM.01043-13.

Lahoz-Monfort JJ., Guillera-Arroita G., Tingley R. 2016. Statistical approaches to account for false-positive errors in environmental DNA samples. Molecular Ecology Resources 16:673685. DOI: $10.1111 / 1755-0998.12486$.

Langlet D., Geslin E., Baal C., Metzger E., Lejzerowicz F., Riedel B., Zuschin M., Pawlowski J., Stachowitsch M., Jorissen FJ. 2013. Foraminiferal survival after long-term in situ experimentally induced anoxia. Biogeosciences 10:7463-7480. DOI: 10.5194/bg-10-74632013.

Lanzén A., Lekang K., Jonassen I., Thompson EM., Troedsson C. 2016. High-throughput metabarcoding of eukaryotic diversity for environmental monitoring of offshore oil-drilling activities. Molecular Ecology 25:4392-4406. DOI: 10.1111/mec.13761.

Laroche O., Wood SA., Tremblay LA., Ellis JI., Lejzerowicz F., Pawlowski J., Lear G., Atalah J., Pochon X. 2016. First evaluation of foraminiferal metabarcoding for monitoring environmental impact from an offshore oil drilling site. Marine Environmental Research 120. DOI: 10.1016/j.marenvres.2016.08.009.

Lau KEM., Washington VJ., Fan V., Neale MW., Lear G., Curran J., Lewis GD. 2015. A novel bacterial community index to assess stream ecological health. Freshwater Biology 60:19882002. DOI: $10.1111 /$ fwb.12625.

Lejzerowicz F., Esling P., Pillet L., Wilding TA., Black KD., Pawlowski J. 2015. Highthroughput sequencing and morphology perform equally well for benthic monitoring of marine ecosystems. Scientific Reports 5:13932. DOI: 10.1038/srep13932.

Logares R., Audic S., Bass D., Bittner L., Boutte C., Christen R., Claverie JM., Decelle J., Dolan JR., Dunthorn M., Edvardsen B., Gobet A., Kooistra WHCF., Mahé F., Not F., Ogata H., Pawlowski J., Pernice MC., Romac S., Shalchian-Tabrizi K., Simon N., Stoeck T., Santini S., Siano R., Wincker P., Zingone A., Richards TA., De Vargas C., Massana R. 2014. Patterns of rare and abundant marine microbial eukaryotes. Current Biology 24:813-821. DOI: $10.1016 /$ j.cub.2014.02.050. 
710 Love MI., Anders S., Huber W. 2014. Differential analysis of count data - the DESeq2 package.

711

712

713

714

715

716

717

718

719

720

721

722

723

724

725

726

727

728

729

730

731

732

733

734

735

736

737

738

739

740

741

742

743

744

745

746

747

748

749

750

751

752

753

754

755 DOI: $110.1186 / \mathrm{s} 13059-014-0550-8$.

Majaneva M., Hyytiäinen K., Varvio SL., Nagai S., Blomster J. 2015. Bioinformatic amplicon read processing strategies strongly affect eukaryotic diversity and the taxonomic composition of communities. PLoS ONE 10:1-18. DOI: 10.1371/journal.pone.0130035.

McConnell H., Calder S., Pannell N., Welsh C. 2014. Maari field development drilling - Marine consent application. Nelson.

McMurdie PJ., Holmes S. 2013. phyloseq: an R package for reproducible interactive analysis and graphics of microbiome census data. PLOS ONE 8:e61217. DOI: 10.1371/journal.pone.0061217.

Mengoni A., Tatti E., Decorosi F., Viti C., Bazzicalupo M., Giovannetti L. 2005. Comparison of 16S rRNA and 16S rDNA T-RFLP approaches to study bacterial communities in soil microcosms treated with chromate as perturbing agent. Microbial ecology 50:375-384. DOI: $10.1007 / \mathrm{s} 00248-004-0222-4$.

Parada AE., Needham DM., Fuhrman JA. 2016. Every base matters: Assessing small subunit rRNA primers for marine microbiomes with mock communities, time series and global field samples. Environmental Microbiology 18:1403-1414. DOI: 10.1111/1462-2920.13023.

Paulson JN., Stine OC., Bravo HC., Pop M. 2013. Differential abundance analysis for microbial marker-gene surveys. Nature Methods 10:1200-1202. DOI: 10.1038/nmeth.2658.

Pawlowski J., Esling P., Lejzerowicz F., Cedhagen T., Wilding TA. 2014. Environmental monitoring through protist NGS metabarcoding: assessing the impact of fish farming on benthic foraminifera communities. Molecular ecology resources 14:early online. DOI: 10.1111/1755-0998.12261.

Pawlowski J., Esling P., Lejzerowicz F., Cordier T., Visco J., Martins C., Kvalvik A., Staven K., Cedhagen T. 2016a. Benthic monitoring of salmon farms in Norway using foraminiferal metabarcoding. Aquaculture Environment Interactions 8:371-386. DOI: 10.3354/aei00182.

Pawlowski J., Lejzerowicz F., Apotheloz-Perret-Gentil L., Visco J., Esling P. 2016b. Protist metabarcoding and environmental biomonitoring: Time for change. European Journal of Protistology 55:12-25. DOI: 10.1016/j.ejop.2016.02.003.

Pawlowski J., Lejzerowicz F., Esling P. 2014. Next-Generation Environmental Diversity Surveys of Foraminifera : Preparing the Future. The Biological Bulletin 227:93-106.

Pochon X., Wood SA., Keeley NB., Lejzerowicz F., Esling P., Drew J., Pawlowski J. 2015. Accurate assessment of the impact of salmon farming on benthic sediment enrichment using foraminiferal metabarcoding. Marine Pollution Bulletin 100:370-382. DOI: 10.1016/j.marpolbul.2015.08.022.

Pochon X., Bott NJ., Smith KF., Wood S a. 2013. Evaluating detection limits of next-generation sequencing for the surveillance and monitoring of international marine pests. PLOS ONE 8:e73935. DOI: 10.1371/journal.pone.0073935.

Quince C., Lanzen A., Davenport RJ., Turnbaugh PJ. 2011. Removing noise from pyrosequenced amplicons. BMC Bioinformatics 12:38. DOI: 10.1186/1471-2105-12-38.

Schafer CT. 2000. Monitoring nearshore marine environments using benthic foraminifera: Some protocols and pitfalls. Micropaleontology 46:161-169.

Schmidt BR., Kéry M., Ursenbacher S., Hyman OJ., Collins JP. 2013. Site occupancy models in the analysis of environmental DNA presence/absence surveys: a case study of an emerging amphibian pathogen. Methods in Ecology and Evolution 4:646-653. DOI: 10.1111/2041210X.12052. 
756 Svarovskaia ES., Cheslock SR., Zhang W-H., Hu W-S., Pathak VK. 2003. Retroviral mutation rates and reverse transcriptase fidelity. Frontiers in bioscience : a journal and virtual library 8:d117-d134. DOI: 10.2741/957.

758

759

760

761

762

763

764

765

766

767

768

769

770

771

772

773

774 Ecology 21:1789-1793. DOI: 10.1111/j.1365-294X.2012.05542.x.

Underwood AJ. 1993. The mechanics of spatially replicated sampling programmes to detect environmental impacts in a variable world. Australian Journal of Ecology 18:99-116. DOI: 10.1111/j.1442-9993.1993.tb00437.x.

Valentini A., Pompanon F., Taberlet P. 2009. DNA barcoding for ecologists. Trends in Ecology and Evolution 24:110-117. DOI: 10.1016/j.tree.2008.09.011.

Visco JA., Apothéloz-Perret-Gentil L., Cordonier A., Esling P., Pillet L., Pawlowski J. 2015. Environmental monitoring: inferring the diatom index from next-generation sequencing data. Environmental Science \& Technology 49:7597-7605. DOI: 10.1021/es506158m.

Wiens JA., Parker KR. 1995. Analyzing the effects of accidental environmental impacts: approaches and assumptions. Ecological Applications 5:1069-1083. DOI: $10.2307 / 2269355$.

Yu DW., Ji Y., Emerson BC., Wang X., Ye C., Yang C., Ding Z. 2012. Biodiversity soup: Metabarcoding of arthropods for rapid biodiversity assessment and biomonitoring. Methods in Ecology and Evolution 3:613-623. DOI: 10.1111/j.2041-210X.2012.00198.x.

Zhan A., Hulák M., Sylvester F., Huang X., Adebayo AA., Abbott CL., Adamowicz SJ., Heath DD., Cristescu ME., Macisaac HJ. 2013. High sensitivity of 454 pyrosequencing for detection of rare species in aquatic communities. Methods in Ecology and Evolution 4:558565. DOI: $10.1111 / 2041-210 X .12037$. 
782

783

784

785

786

787

788

789

790

791

792

793

794

795

796

797

798

799

800

801

802

803

804

805

806

807

808

809

810

811

812

813

814

815

816

817

818

819

820

821

822

823

824

825

\section{List of figures}

Figure 1. Site map indicating: A) The study site situated in the Taranaki Bight (Maari facility, black circle); B) The Floating Production Storage and Offloading (FPSO) platform. Sampling stations are in full circle, with numbers corresponding to the distance to the platform along the major water flow axis. Modified from Johnston et al. (2014).

Figure 2. Venn diagrams displaying shared and unshared operational taxonomic units (OTUs) among environmental DNA (eDNA) and RNA (eRNA) amplicons of the bacteria (A, B) and eukaryote $(C, D)$ datasets.

Untrimmed data are represented in $\mathbf{A}$ and $\mathbf{C}$ datasets, with the removed singletons shown in $\mathbf{B}$ and $\mathbf{D})$.

Figure 3. Relative abundance of the ten most important bacterial classes (A) and eukaryotic phyla (B) removed during the trimmed by shared OTUs method.

Abundance values for each class/phylum are stacked in order from greatest to least, separated by a thin horizontal line. eDNA = environmental DNA, eRNA = environmental RNA.

Figure 4. Non-metric multidimensional scaling (nMDS) plots.

A) Trimmed by singletons and B) trimmed by shared operational taxonomic units (OTUs) data of both environmental DNA (eDNA) and RNA (eRNA) of bacteria (left) and eukaryotes (right). $\mathrm{NF}=$ near-field; $\mathrm{FF}=$ far-field.

\section{List of tables}

Table 1. Number of operational taxonomic units (OTUs) and reads per dataset.

Brackets show portion of reads removed by each technique from the raw dataset. SD = Standard deviation, eDNA = environmental DNA, and eRNA = environmental RNA.

Table 2. Non-parametric t-tests on alpha-diversity metrics on observed operational taxonomic units (OTUs) and Shannon index between near field stations $(\leq 250 \mathrm{~m})$ and far field stations $(>$ $250 \mathrm{~m})$.

Based on the data kept after the different trimming methodologies. eDNA = environmental DNA, eRNA = environmental RNA. Significant $\mathrm{p}$-values are in bold.

Table 3. Adonis and Mantel tests.

Analysis of the strength and statistical significance of sample groupings among datasets, and correlations between beta-diversity matrices of micro (bacteria and eukaryotes) and macro-fauna, and with distance matrix of selected environmental variables. eDNA = environmental DNA, eRNA = environmental RNA. Significant p-values are in bold.

\section{List of supplementary figures}


826 Figure S1. Rarefaction curves on Chao 1 and Shannon metrics for each of the 18 environmental

827 DNA and RNA amplicons of bacteria (A) and eukaryotes (B) analyzed using high-throughput

828 sequencing.

829

830

831

832

833

834

835

836

837

838

839

840

841

842

843

844

845

846

847

848

849

850

851

852

853

854

855

856

857

858

859

860 Table S9. Distance-based test for homogeneity of multivariate dispersions (Permdisp) analysis

861 with 999 permutations, assessing the beta-diversity variance between biological replicates.

862 Deviations are from centroids. Significant p-values are in bold. 
Figure 1

Site map indicating: A) The study site situated in the Taranaki Bight (Maari facility, black circle); B) The Floating Production Storage and Offloading (FPSO) platform.

Sampling stations are in full circle, with numbers corresponding to the distance to the platform along the major water flow axis. Modified from Johnston et al. (2014).



B

Major flow axis

dominant current direction



S1000 


\section{Figure 2 (on next page)}

Venn diagrams displaying shared and unshared operational taxonomic units (OTUs) among environmental DNA (eDNA) and RNA (eRNA) amplicons of the bacteria (A, B) and eukaryote $(C, D)$ datasets.

Untrimmed data are represented in $\mathbf{A}$ and $\mathbf{C}$ datasets, with the removed singletons shown in B and D). 


\section{A)}
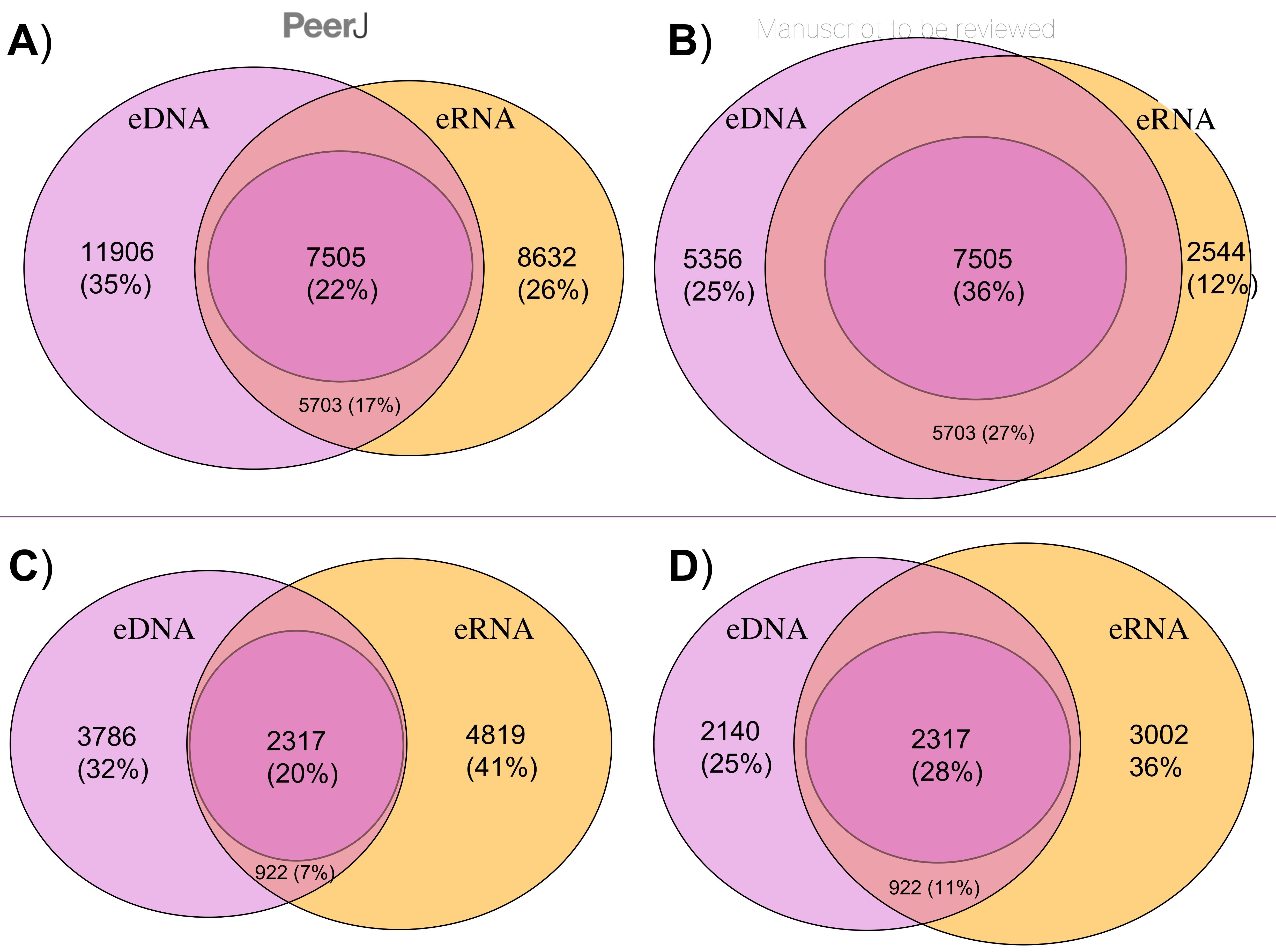

eDNA OTUs only OTUs shared between eDNA/eRNA datasets (distinct samples) eRNA OTUs only Peerr reviewhing PDF K2017:01:15580:1:1:NEW 20 Mar 2017) 
Figure 3 (on next page)

Relative abundance of the ten most important bacterial classes (A) and eukaryotic phyla (B) removed during the trimmed by shared OTUs method.

Abundance values for each class/phylum are stacked in order from greatest to least, separated by a thin horizontal line. eDNA = environmental DNA, eRNA = environmental RNA. 
A)

ó

eDNA

60

40

20.

0 .

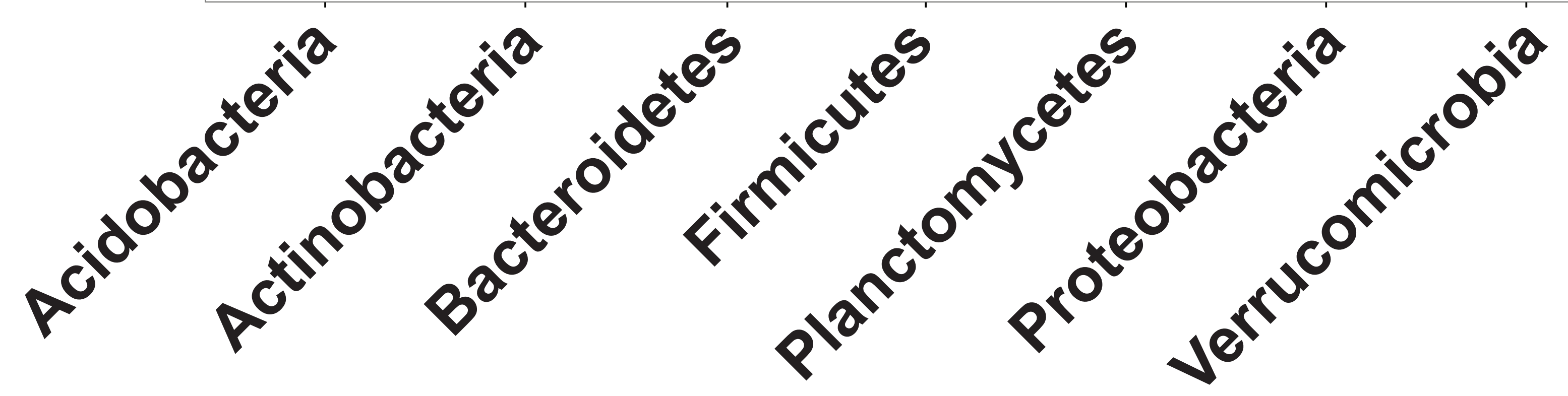

B)

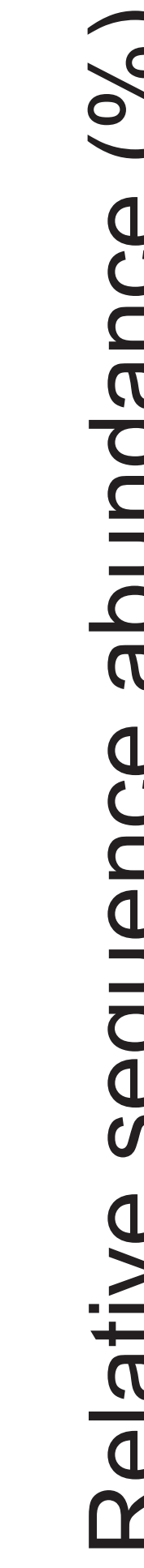

0.

60

eDNA

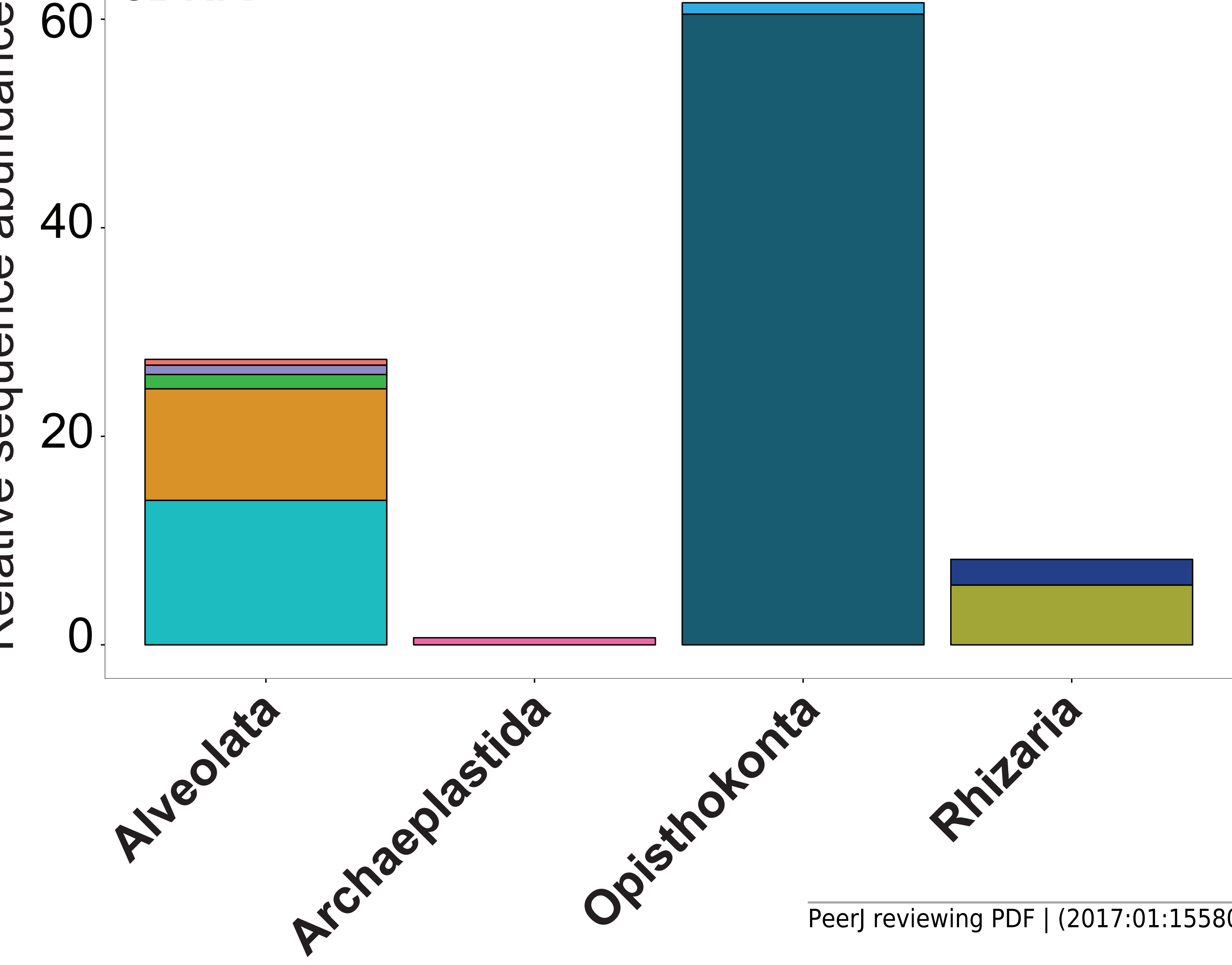

PeerJ

Manuscript to be reviewed

\section{eRNA}

60

40

20

0

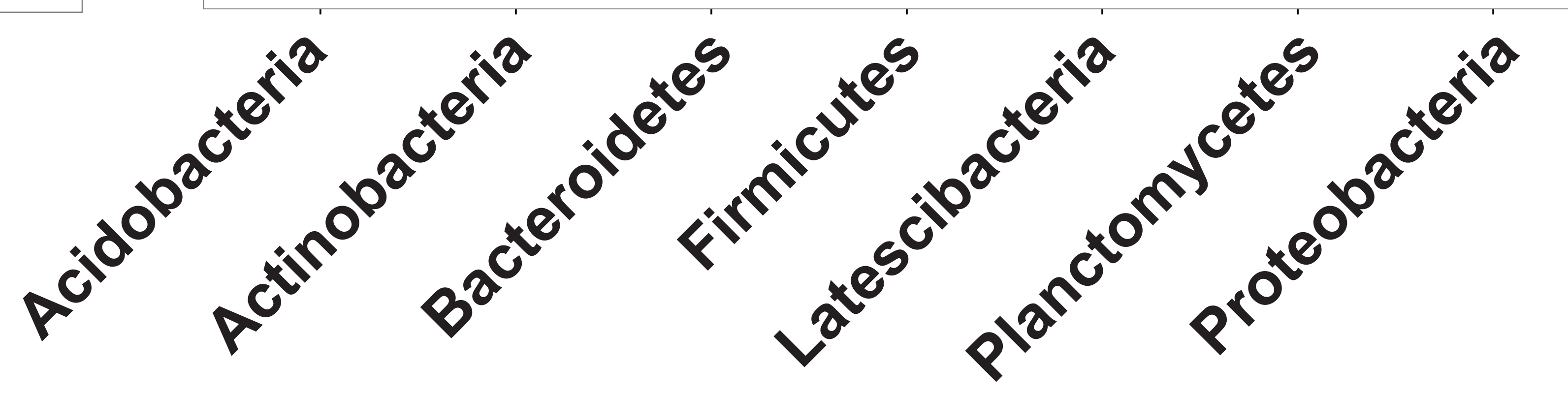

\section{$i^{2}$}

.

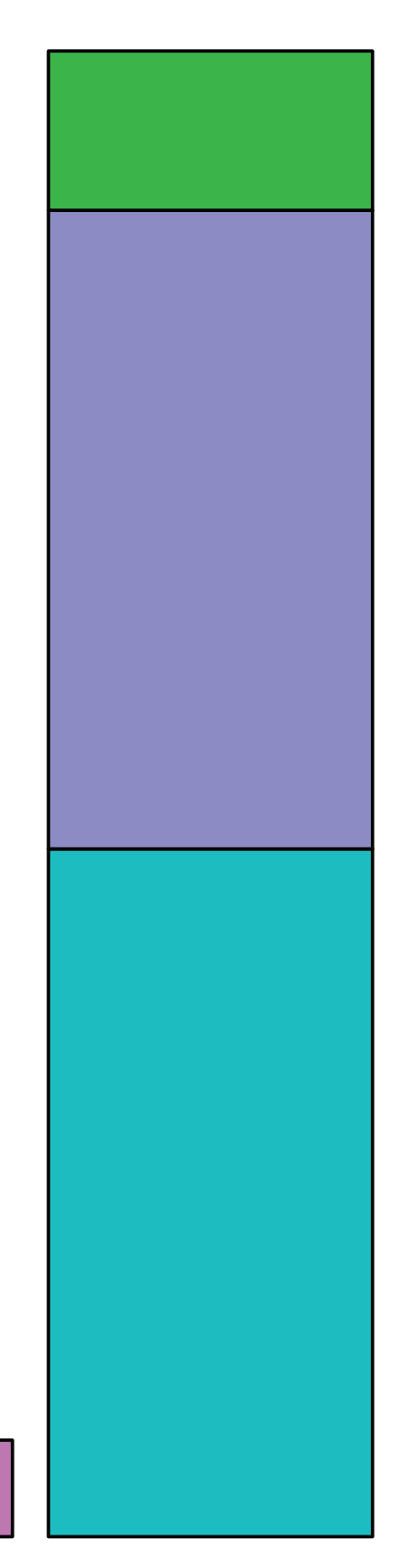

Acidobacteria_Gp10

Acidobacteria_Gp22

Actinobacteria

Alphaproteobacteria

Clostridia

Deltaproteobacteria

Flavobacteria

Gammaproteobacteria

Latescibacteria

Planctomycetia

Verrucomicrobiae
60. eRNA

40

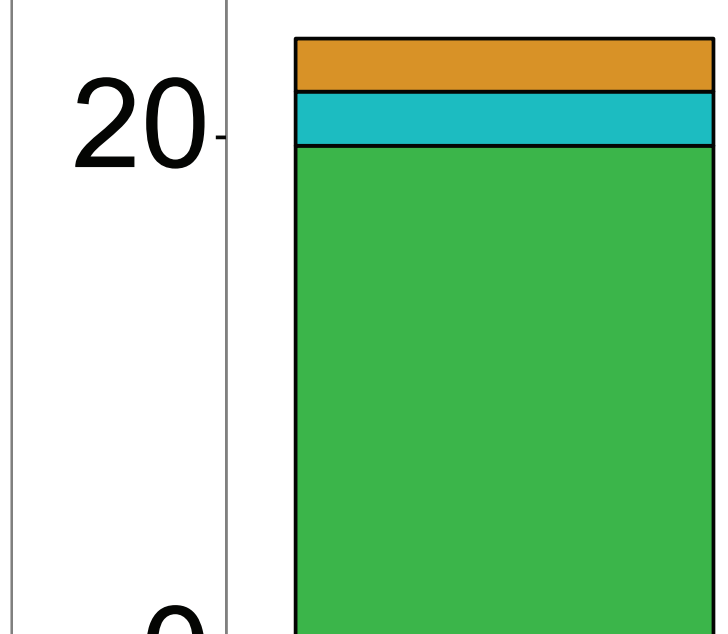

0

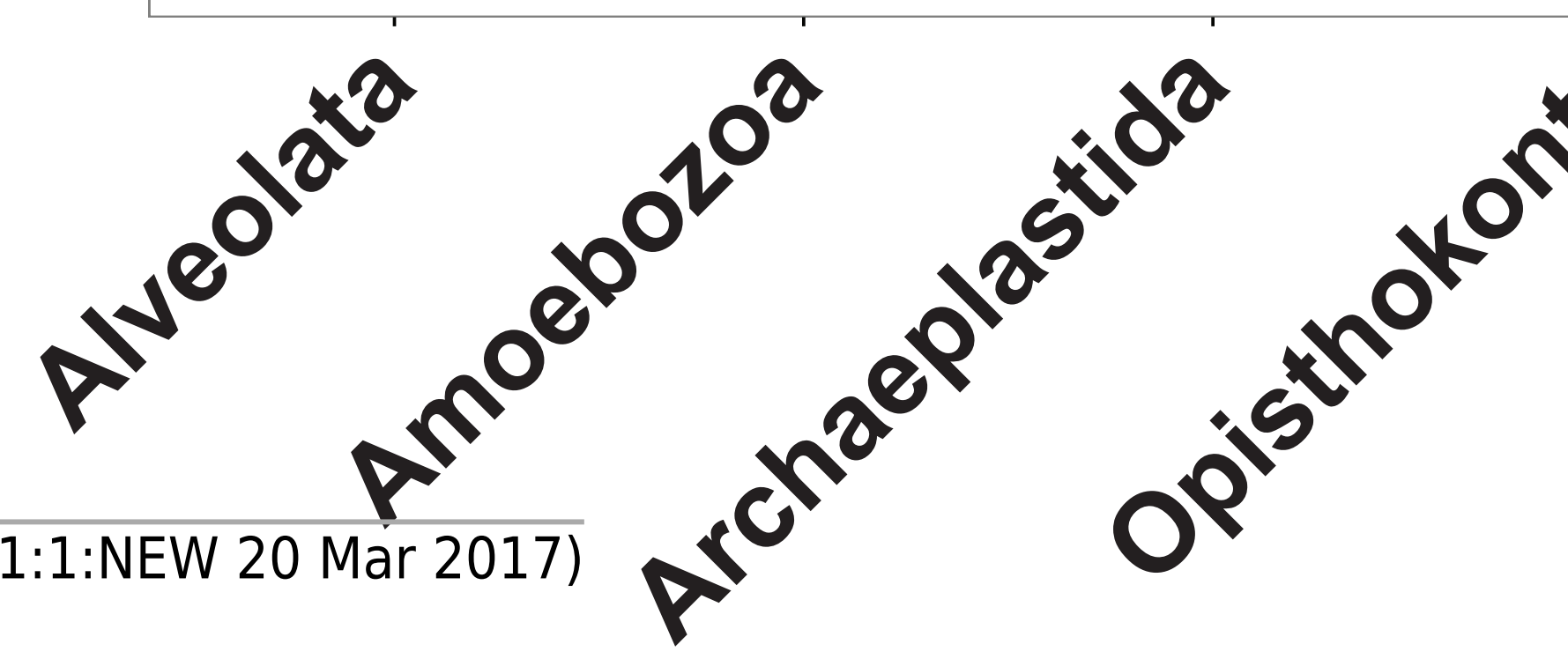

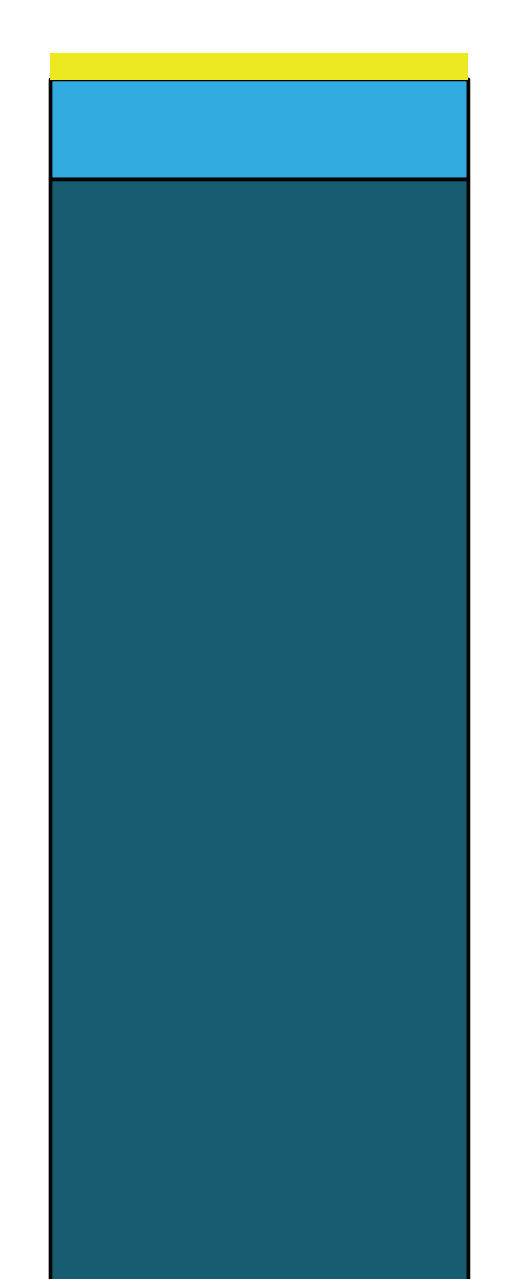


Alveolata

Amoebozoa

Apicomplexa

Cercozoa

Choanoflagellida

Ciliophora

Dinophyta

Fungi

Metazoa

Ochrophyta

Perkinsea

Radiolaria

Streptophyta 


\section{Figure 4(on next page)}

Non-metric multidimensional scaling (nMDS) plots.

A) Trimmed by singletons and B) trimmed by shared operational taxonomic units (OTUs) data of both environmental DNA (eDNA) and RNA (eRNA) of bacteria (left) and eukaryotes (right). $\mathrm{NF}=$ near-field; FF $=$ far-field . 
Pegalteria

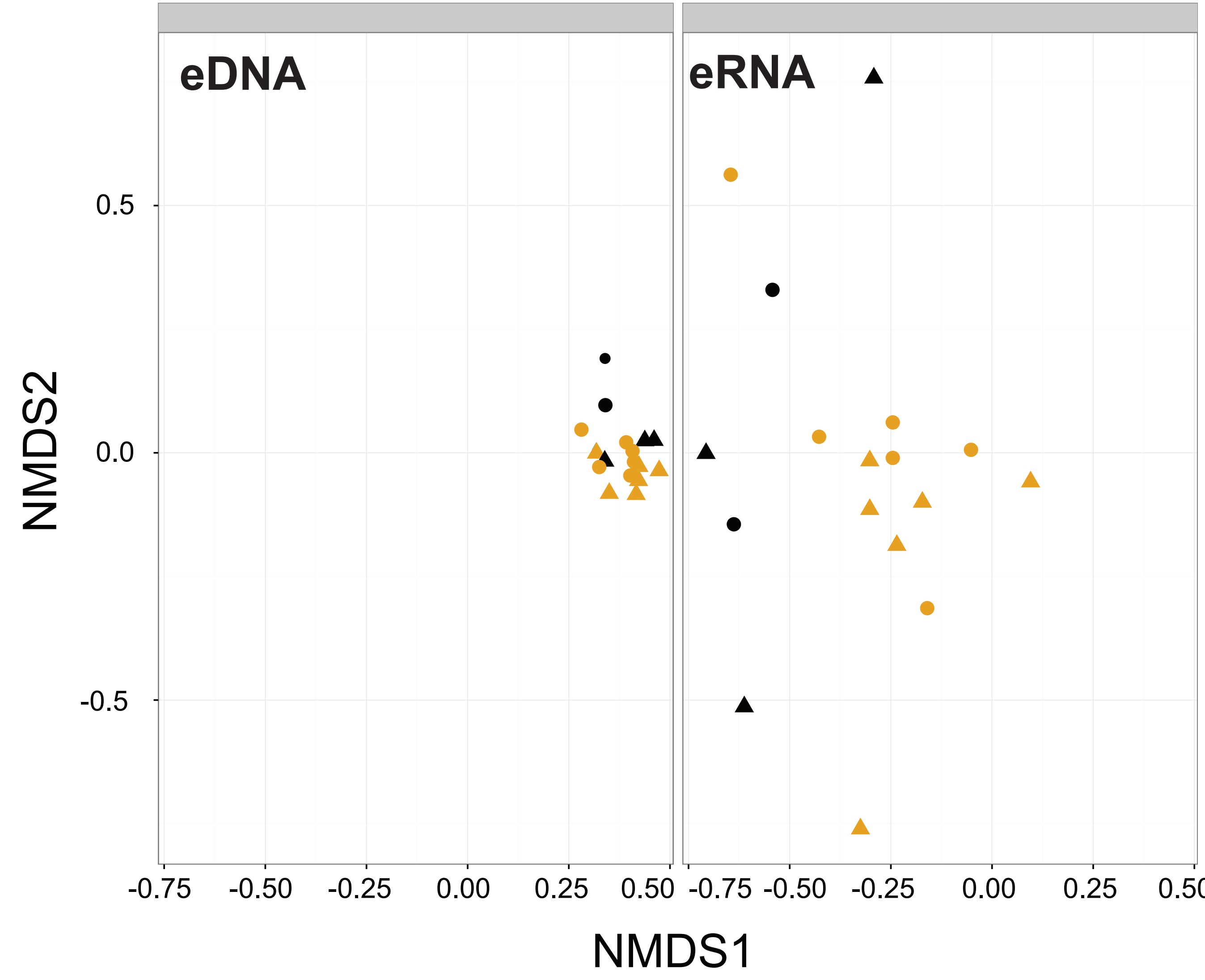

B)

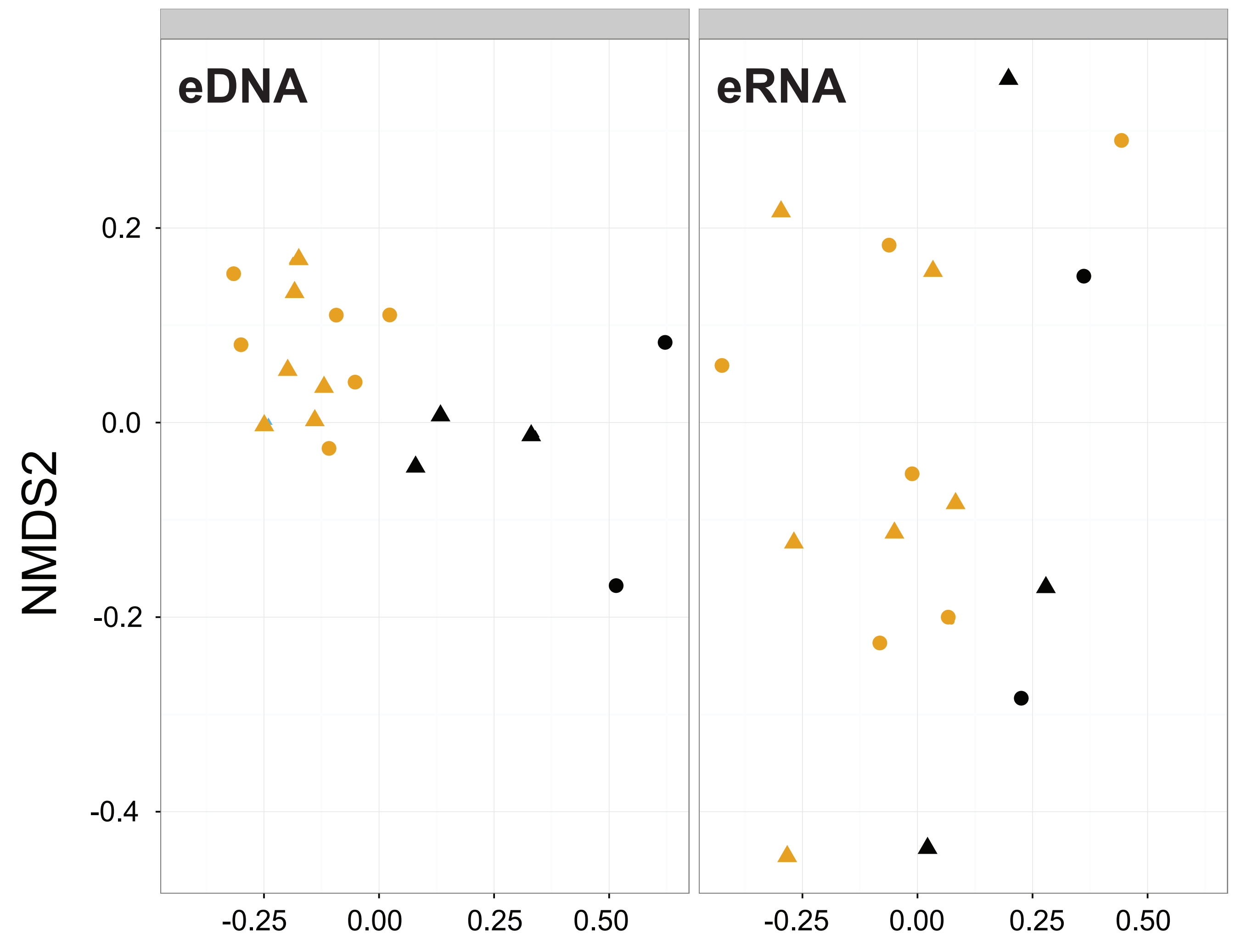

Bacteria

RePMUDiesing PDF I (2017:01:15580:1:1:NEW 20 Mar 2017)
Manuscript Eukaryotesd

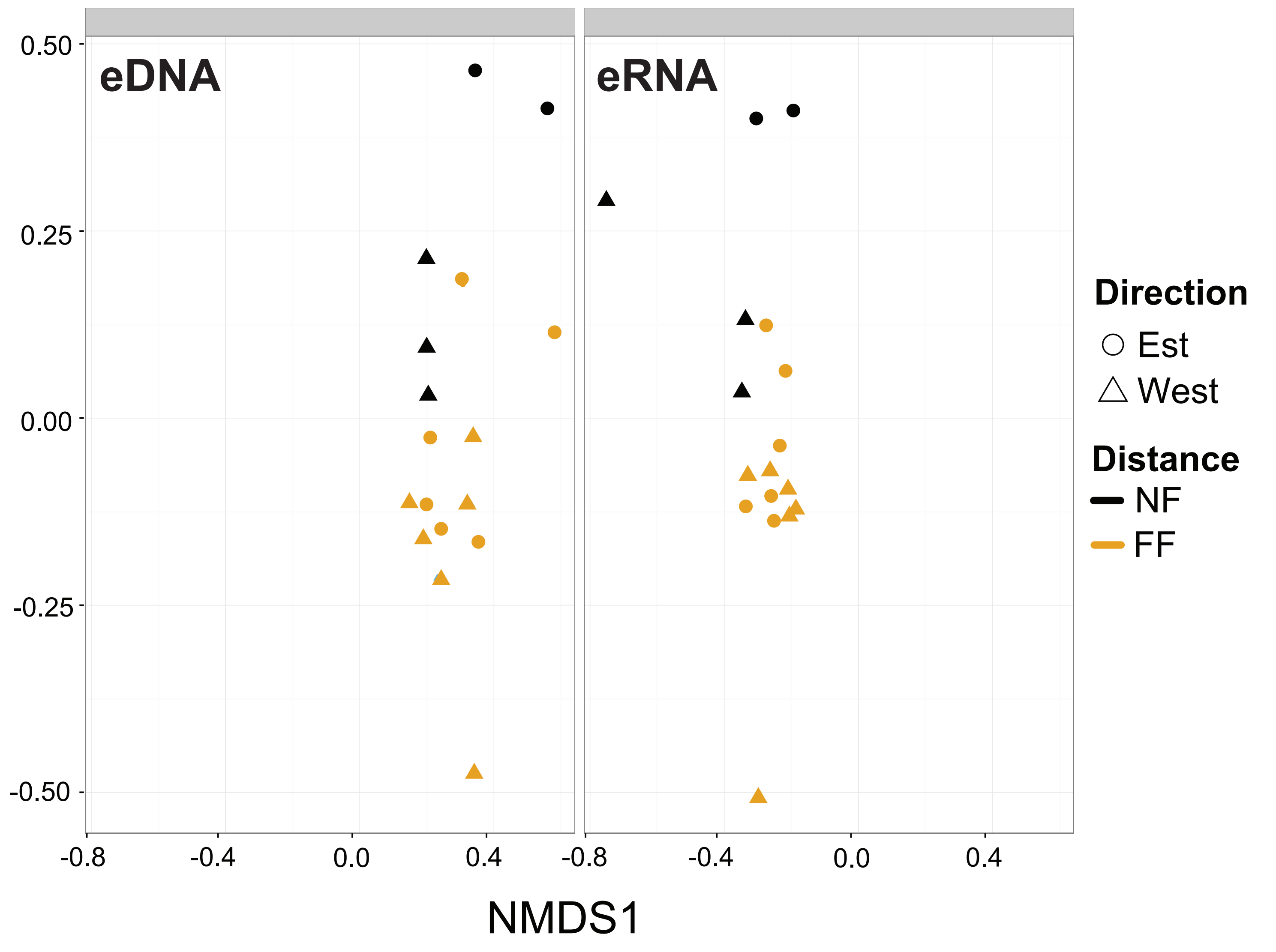

Eukaryotes

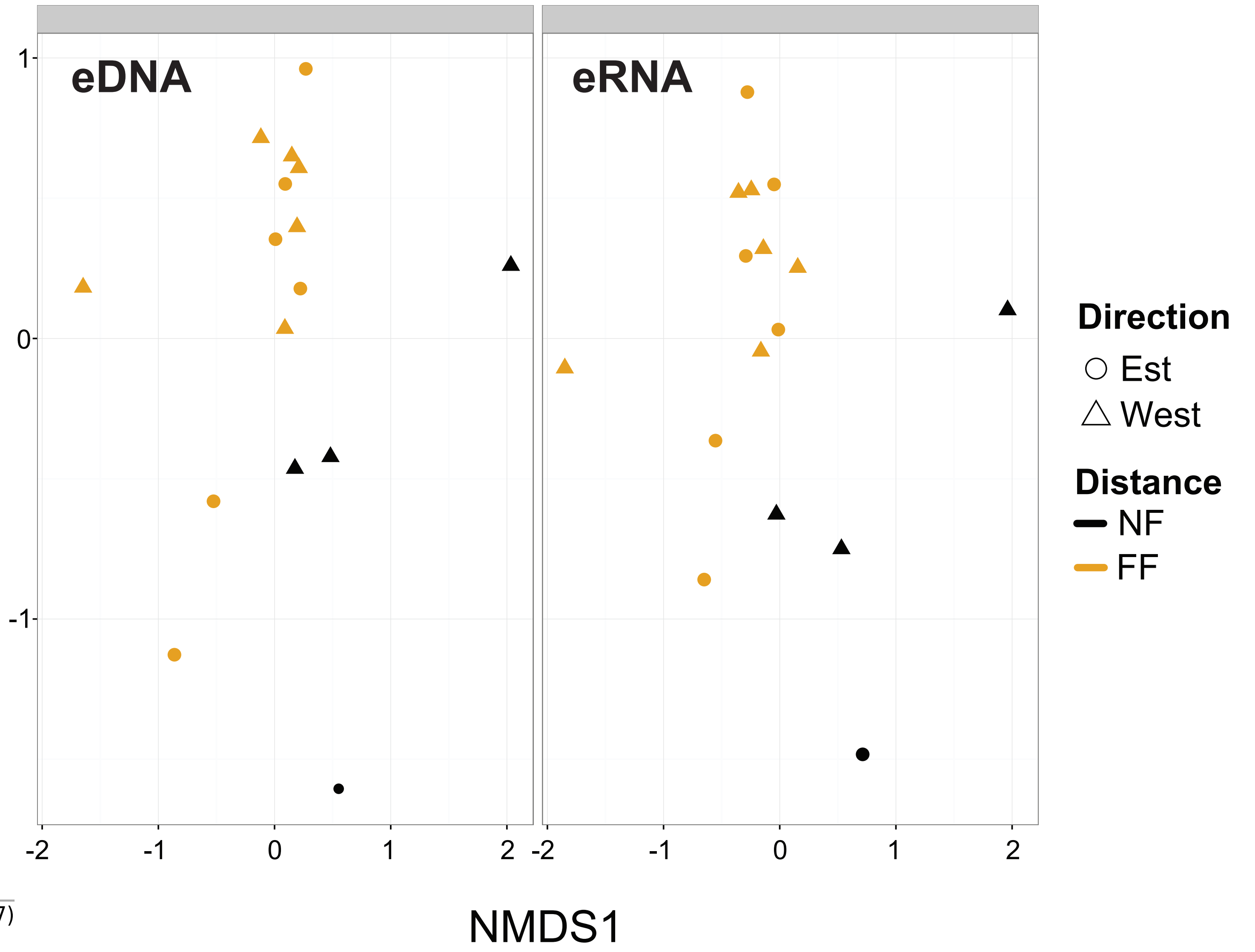




\section{Table $\mathbf{1}$ (on next page)}

Number of operational taxonomic units (OTUs) and reads per dataset.

Brackets show portion of reads removed by each technique from the raw dataset. SD = Standard deviation, eDNA = environmental DNA, and eRNA = environmental RNA. 
1

2

3

4 5

6

7

\begin{tabular}{|c|c|c|c|c|c|c|c|c|}
\hline & Trimming technique & Total & $\begin{array}{r}\text { Reads } \\
\text { Mean number of } \\
\text { sequence reads } \\
\text { per removed } \\
\text { OTUs [SD] }\end{array}$ & $\begin{array}{c}\text { eDNA } \\
\text { dataset }\end{array}$ & $\begin{array}{c}\text { eRNA } \\
\text { dataset }\end{array}$ & Total & $\begin{array}{l}\text { eDNA } \\
\text { dataset }\end{array}$ & $\begin{array}{c}\text { eRNA } \\
\text { dataset }\end{array}$ \\
\hline \multirow{3}{*}{ 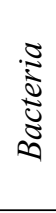 } & Raw data & 871557 & NA & 529180 & 342377 & 33746 & 25114 & 21840 \\
\hline & Trimmed by singletons & $\begin{array}{c}858919 \\
(1 \%)\end{array}$ & NA & 522630 & 336289 & $\begin{array}{l}21108 \\
(37 \%)\end{array}$ & 18564 & 15752 \\
\hline & Trimmed by shared OTUs & $\begin{array}{c}633643 \\
(27 \%)\end{array}$ & $3.1[15.7]$ & 348860 & 284783 & $\begin{array}{c}7505 \\
(78 \%)\end{array}$ & 7505 & 7505 \\
\hline \multirow{3}{*}{ 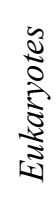 } & Raw data & 767855 & NA & 414271 & 353584 & 11844 & 7025 & 8058 \\
\hline & Trimmed by singletons & $\begin{array}{l}764392 \\
(0.4 \%)\end{array}$ & NA & 412625 & 351767 & $\begin{array}{c}8381 \\
(29 \%)\end{array}$ & 5379 & 6241 \\
\hline & Trimmed by shared OTUs & $\begin{array}{c}553651 \\
(28 \%) \\
\end{array}$ & $8.6[99]$ & 308285 & 245366 & $\begin{array}{c}2317 \\
(80 \%) \\
\end{array}$ & 2317 & 2317 \\
\hline
\end{tabular}

Table 1: Number of operational taxonomic units (OTUs) and reads per dataset. Brackets show portion of reads removed by each technique from the raw dataset. SD = Standard deviation, eDNA = environmental DNA, and eRNA = environmental RNA. 


\section{Table 2 (on next page)}

Non-parametric t-tests on alpha-diversity metrics on observed operational taxonomic units (OTUs) and Shannon index between near field stations $(\leq 250 \mathrm{~m}$ ) and far field stations (> $250 \mathrm{~m}$ ).

Based on the data kept after the different trimming methodologies. eDNA = environmental DNA, eRNA = environmental RNA. Significant $p$-values are in bold. 
2

3

4 Table 2: Non-parametric t-tests on alpha-diversity metrics observed Operational Taxonomic

5 Units (OTUs) and Shannon index between near field stations $(\leq 250 \mathrm{~m})$ and far field stations $(>$ $6250 \mathrm{~m}$ ), on the data kept after the different trimming methodologies. eDNA = environmental

7 DNA, eRNA = environmental RNA. Significant p-values are in bold. 8

\begin{tabular}{|c|c|c|c|c|c|c|c|c|c|c|}
\hline \multirow{2}{*}{\multicolumn{3}{|c|}{ Datasets }} & \multicolumn{4}{|c|}{ Observed OTUs } & \multicolumn{4}{|c|}{ Shannon index } \\
\hline & & & $\begin{array}{l}\text { Near- } \\
\text { field }\end{array}$ & $\begin{array}{l}\text { Far- } \\
\text { field }\end{array}$ & $t$-stat & $p$-value & $\begin{array}{l}\text { Near- } \\
\text { field }\end{array}$ & $\begin{array}{l}\text { Far- } \\
\text { field }\end{array}$ & $t$-stat & $p$-value \\
\hline \multirow{4}{*}{ 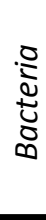 } & \multirow{2}{*}{$\begin{array}{l}\text { Trimmed by } \\
\text { singletons }\end{array}$} & eDNA & 2306 & 2348 & -0.33 & 0.71 & 9.6 & 9.83 & -1.07 & 0.32 \\
\hline & & eRNA & 1274 & 1495 & -2.08 & 0.06 & 7.86 & 8.05 & -0.82 & 0.43 \\
\hline & \multirow{2}{*}{$\begin{array}{l}\text { Trimmed by } \\
\text { shared OTUs }\end{array}$} & eDNA & 807 & 1356 & -6.24 & $<0.01$ & 7.56 & 8.63 & -5.04 & $<0.01$ \\
\hline & & eRNA & 958 & 1111 & -1.57 & 0.13 & 7.25 & 7.42 & -0.79 & 0.44 \\
\hline \multirow[b]{4}{*}{4} & \multirow{2}{*}{$\begin{array}{l}\text { Trimmed by } \\
\text { singletons }\end{array}$} & eDNA & 550 & 572 & -0.30 & 0.77 & 6.75 & 7.06 & -0.77 & 0.48 \\
\hline & & eRNA & 686 & 830 & -1.91 & 0.07 & 7.05 & 7.47 & -1.05 & 0.29 \\
\hline & \multirow{2}{*}{$\begin{array}{l}\text { Trimmed by } \\
\text { shared OTUs }\end{array}$} & eDNA & 277 & 415 & -2.16 & 0.04 & 5.78 & 6.45 & -1.62 & 0.13 \\
\hline & & eRNA & 326 & 432 & -1.42 & 0.17 & 5.67 & 6.42 & -1.56 & 0.14 \\
\hline
\end{tabular}

9 


\section{Table 3(on next page)}

Adonis and Mantel tests.

Analysis of the strength and statistical significance of sample groupings among datasets, and correlations between beta-diversity matrices of micro (bacteria and eukaryotes) and macrofauna, and with distance matrix of selected environmental variables. eDNA = environmental DNA, eRNA = environmental RNA. Significant $p$-values are in bold. 
2

3 Table 3: Analysis of the strength and statistical significance of sample groupings among 4 datasets, and correlations between beta-diversity matrices of micro (bacteria and eukaryotes) and 5 macro- fauna, and with distance matrix of selected environmental variables. eDNA =

6 environmental DNA, eRNA = environmental RNA. Significant p-values are in bold.

\begin{tabular}{|c|c|c|c|c|c|c|c|c|c|c|}
\hline \multicolumn{3}{|c|}{ Datasets } & \multicolumn{2}{|c|}{$\begin{array}{l}\text { Adonis } \\
\text { Near-field VS } \\
\text { Far-field }\end{array}$} & \multicolumn{2}{|c|}{$\begin{array}{l}\text { Correlation with } \\
\text { macro-fauna }\end{array}$} & \multicolumn{2}{|c|}{$\begin{array}{l}\text { Mantel test } \\
\text { Correlation with } \\
\text { env. variables }\end{array}$} & \multicolumn{2}{|c|}{$\begin{array}{l}\text { Correlation } \\
D N A / R N A\end{array}$} \\
\hline & & & $\mathrm{R}^{2}$ & $\mathrm{p}$-value & $r$ & $p$-value & $r$ & $p$-value & $r$ & $\mathrm{p}$-value \\
\hline \multirow{4}{*}{  } & \multirow{2}{*}{ Trimmed by singletons } & $e D N A$ & 0.07 & 0.01 & -0.53 & $<0.01$ & 0.52 & $<0.01$ & \multirow[b]{2}{*}{-0.05} & \multirow{2}{*}{0.80} \\
\hline & & eRNA & 0.07 & 0.21 & -0.06 & 0.78 & 0.04 & 0.82 & & \\
\hline & \multirow{2}{*}{$\begin{array}{l}\text { Trimmed by shared } \\
\text { OTUs }\end{array}$} & $e D N A$ & 0.10 & $<0.01$ & -0.51 & $<0.01$ & 0.58 & $<0.01$ & \multirow{2}{*}{0.31} & \multirow{2}{*}{0.08} \\
\hline & & eRNA & 0.07 & 0.01 & -0.08 & 0.73 & 0.08 & 0.63 & & \\
\hline \multirow{4}{*}{ 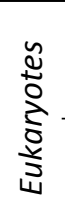 } & \multirow{2}{*}{ Trimmed by singletons } & $e D N A$ & 0.08 & 0.04 & -0.35 & 0.05 & 0.52 & $<0.01$ & \multirow{2}{*}{0.47} & \multirow{2}{*}{0.02} \\
\hline & & eRNA & 0.12 & $<0.01$ & -0.14 & 0.48 & 0.24 & 0.09 & & \\
\hline & \multirow{2}{*}{$\begin{array}{l}\text { Trimmed by shared } \\
\text { OTUs }\end{array}$} & eDNA & 0.10 & 0.02 & $<0.01$ & 0.99 & 0.32 & 0.02 & \multirow{2}{*}{0.89} & \multirow{2}{*}{$<0.01$} \\
\hline & & eRNA & 0.11 & $<0.01$ & -0.09 & 0.67 & 0.18 & 0.30 & & \\
\hline
\end{tabular}

7 